\title{
C-A/AP/\#411
}

Nov. 2010

\section{A Simple Crunching of the AGS "bare" machine ORM Data - February 2007 - to extract some aspects of AGS Transverse Coupling at Injection and Extraction}

\author{
L. Ahrens
}

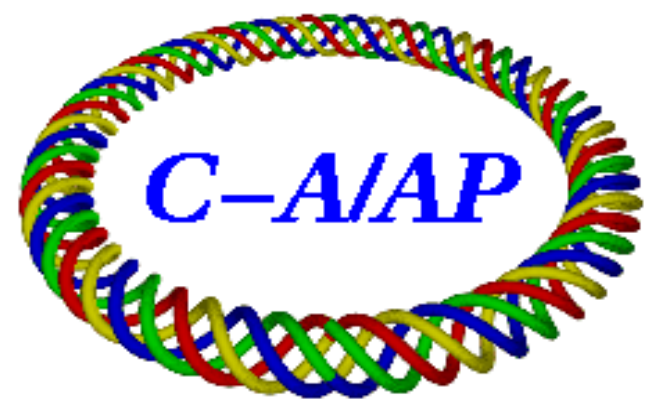

\section{Collider-Accelerator Department Brookhaven National Laboratory Upton, NY 11973}




\section{A Simple Crunching of the AGS "bare" machine ORM Data - February 2007 - to extract some aspects of AGS Transverse Coupling at Injection and Extraction}

\section{Leif Ahrens July 2010 _ December 2009}

\section{Acknowledgements:}

Just because most readers will not get to the end: Operations, lead by Vincent Schoefer collected this data. Vincent organized it for us. J.W.Glenn pointed out one symmetry in the data which let to the deeper digging given here.

\section{Introduction and some definitions:}

The objective of this note is to (once again) explore the AGS "ORM" (orbit response matrix) data taken (by Operations) early during the 2007 run with an AGS bare machine and gold beam. Indeed the present motivation is to extract as much information about the AGS inherent transverse coupling as possible - from general arguments and the copious ORM data. And taking this one step further, (though not accomplished yet) the goal really should be to tell the model how to describe this coupling.

"Bare" as used here means the AGS with no quadrupole, sextupole or octupole magnets powered. Only the main (combined-function) magnet string and dipole bumps necessary to optimize beam survival are powered. "ORM data" means the systematic recording of the equilibrium orbit beam position monitor response to powering individual dipole corrector magnets. The "matrix" results from looking at the effect of each of the (12 superperiods X 4 dipoles per superperiod) "kicks" on each of the $(12 \times 6)$ pick up electrodes (pues) in each transverse plane. So then we have two (48X72) matrices of numbers from the ORM data. (Though "pue" usually refers to the hardware in the vacuum chamber and "bpm" to the beam position monitoring system, the two labels will be used casually here.)

The exercise is carried out at two magnet rigidities, injection (AGS field $\sim 434$ Gauss) and extraction to RHIC ( $\sim 9730$ Gauss), - a ratio of rigidities of about 22.4. Since we stick with a bare machine, we are also stuck with the bare tunes which means the tunes are rather close together and near 8.75. (Injection : $(\mathrm{h}, \mathrm{v}) \sim$ $(8.73,8.76)$.

\section{The Analysis Plan:}

To extract information from the data requires a model. Here that model starts with the assumption of a superperiod symmetric machine (one reason to use a bare machine) and with correctors and pues that are assumed perfect. The first game is to use the superperiodic assumption to identify "bad" pues and correctors since a superperiodic machine will give the same response if both the introduced distortion - the added dipole - and the pickups are shifted by any number of 
superperiods. By playing the superperiodicity card we get twelve orbits around the machine that should all be identical. We take the average of these twelve (shifted) orbits at each pue to be the best estimate of the true orbit and look for inconsistant pues. Our assumption has reduced the (48x72) data points down to $4 \times(1 \times 72)$. Each of these 72 orbit locations, defined by their phase (or distance) from the active kick, contains the average response and the individual response from the 12 correctors located at a particular superperiod position (e.g. a \#2 ss) and each location along the orbit gets contributions from a pue in each superperiod. Of course the 12 numbers at a given pue phase location are not identical but their variation should just reflect the inherent error in the pues and should be the same for all 72. On the first pass we find this to not be true. There are large variations in the resulting standard deviations from one orbit location to another. A visual analysis of these data identifies two horizontal and four vertical pues whose responses wander far from the rest. (Indeed two of these are flagged in the data set as very questionable). We exclude all of these from the data set. We proceed then under the revised assumption that pues are either bad or good and we have dumped the bad horizontal and vertical and on the basis that the "in plane" analysis should be "superperiodic" in the sense described above. And even with this cutting we still have plenty of points contributing to the average orbits. (This process of throwing away bad data could be done more systematically - but still using this measure.)

With this data set we should get a confirmation of the superperiodicity of the machine - in particular applied to the beta functions at the kick and at the pues - and applied to the strength of the kicks i.e. the orbits from for example the various \#2 kicks, when shifted in plotting to start at the kick, overlay well. We can also calculate explicitly (if we know the kick strength and the betatron tune) the beta function at the kick from the orbit shift measured there. And here we would get the superperiod symmetric evaluation and an error estimate on that evaluation.

The next step is to look at the response in the unkicked plane, having frozen the selection of "bad" electrodes based on the high quality in-plane responses. Now the specific cause for any equilibrium orbit motion - as individual correctors in the other plane are powered - is not known. So the assumption of invariance under superperiod shifting is not required. Indeed apriori it is not even expected since that would require that the source for the coupling was itself superperiodic symmetric. The interpretation of the results as model modifications is not so trivial and not found here, but some strongly constraining aspects are of the coupling behavior are evident.

\section{The data - results at gold injection - first kicks in the horizontal plane then vertical:}

So now we look at the data starting with injection. Here the available corrector strengths are large enough to exceed the machine aperture which means we can get optimal signal to noise. A 4 ampere excitation fits the aperture. (The 
corrector dipole power supplies have a maximum current of $25 \mathrm{amps}$ ). The result in the horizontal pues around the ring for the kicks at the \#8 horizontal correctors is shown in figure 1. All the injection data is presented in appendix I.

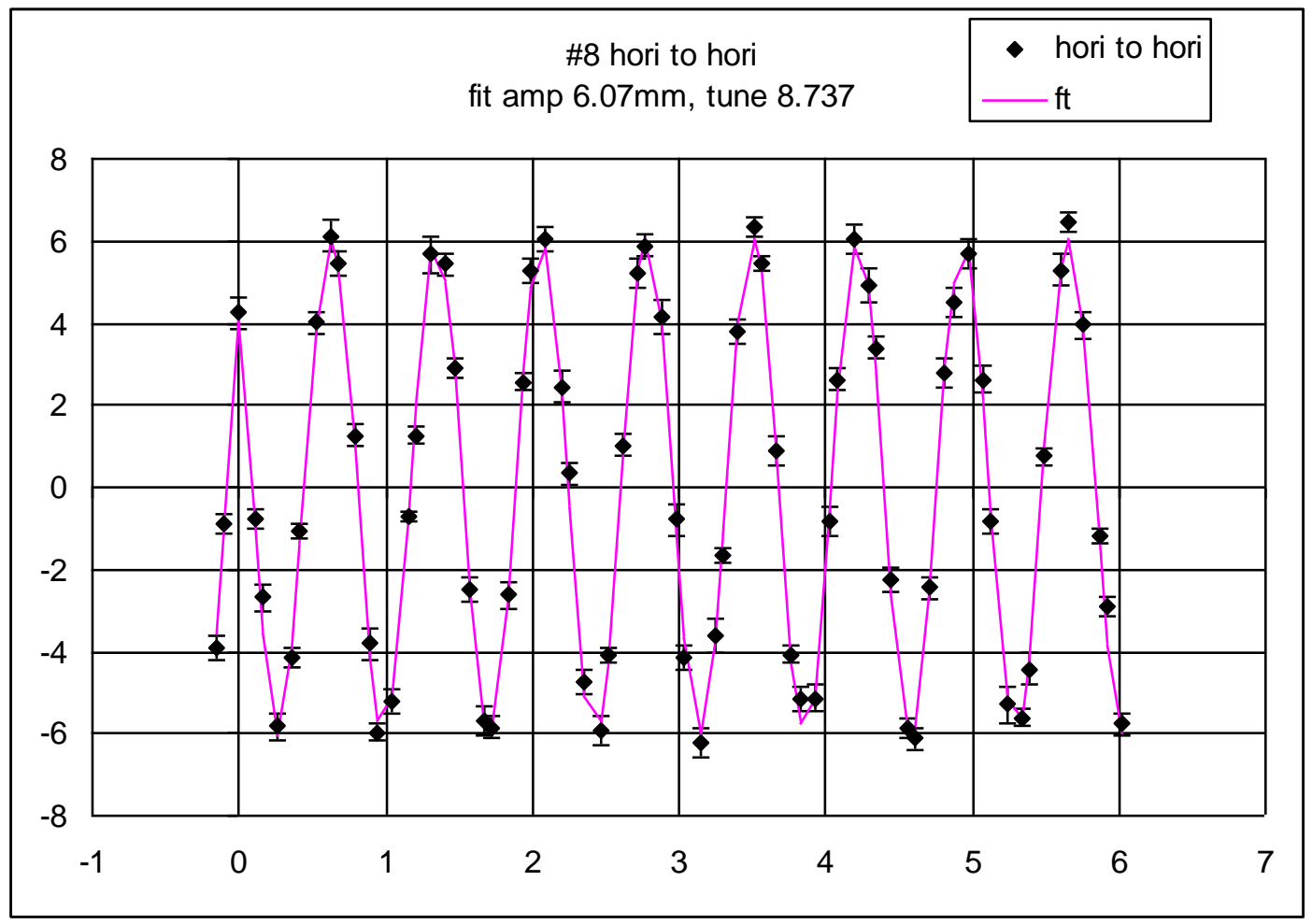

Figure 1 Average horizontal equilibrium orbit response to powering a horizontal dipole corrector at the \#8 straight section - vertical axis: orbit change in $\mathrm{mm}$, horizontal axis: phase "geometric" around the ring starting at the kick. The "fit" is described later.

The response in the kicked plane is - not surprisingly - consistent with the assumptions made above and suggests even stronger ones (which are due to the approximate 60 fold symmetry of the AGS lattice and are consistent with the standard more detailed model for the AGS). All the \#8 kicks measured through the shifting pues give the same orbit to within the standard deviation error bars shown. These standard deviations are about the same for all of the orbit locations and here the average is $0.3 \mathrm{~mm}$ with a standard deviation (of these standard deviations now stepping from the kick around the ring) of $.07 \mathrm{~mm}$. These two numbers - average and standard deviation - are essentially the same for the set of horizontal kicks at 2 , 8,12 , or 18 .

The amplitudes for the resulting oscillations are also nearly the same independent of the dipole powered. This goes beyond the superperiodic assumption. The orbit amplitude number is $\sim 6 \mathrm{~mm}$ for all four kicks (see table 1 ). The kick currents are always set to be the same so this observation is consistent with assuming the beta functions at the pues and at the kick are nearly equal as we 
move from kicking at \#2 to \#8, \#12, and \#18. The bare MAD model expects this. An AGS with 60 fold superperiodicity would have this.

\begin{tabular}{|c|c|c|c|c|c|c|}
\hline \multirow[b]{2}{*}{$\begin{array}{l}\text { powered } \\
\text { dipole \# }\end{array}$} & \multicolumn{2}{|c|}{ superperiodicity } & \multicolumn{2}{|l|}{$\begin{array}{l}\text { simple } \\
\text { fit }\end{array}$} & \multicolumn{2}{|c|}{$\mathrm{H}$ pos at Kick } \\
\hline & $\begin{array}{l}\text { sigma pue } \\
\mathrm{mm}\end{array}$ & $\begin{array}{l}\mathrm{sd} \\
\mathrm{mm}\end{array}$ & $\begin{array}{l}\text { Amp } \\
\mathrm{Mm}\end{array}$ & Tune & $\begin{array}{l}\mathrm{sp} \text { ave } \\
\mathrm{mm}\end{array}$ & $\begin{array}{l}\text { sigma } \\
\mathrm{mm}\end{array}$ \\
\hline 2 & 0.32 & 0.07 & 6.1 & 8.734 & 4.11 & 0.32 \\
\hline 8 & 0.30 & 0.07 & 6.07 & 8.737 & 4.24 & 0.40 \\
\hline 12 & 0.30 & 0.06 & 5.87 & 8.736 & 3.75 & 0.28 \\
\hline 18 & 0.35 & 0.07 & 5.97 & 8.733 & 3.76 & 0.33 \\
\hline
\end{tabular}

Table 1 horizontal response to horizontal dipole at injection (au)

In the above table the first column lists the straight section of the powered horizontal dipole. The second and third give the just mentioned variation from pues at the same phase from the kick, and then of this variation going around the ring. The 4th and 5th are from a simple fit to the data of a cosine wave having its origin opposite the kick - and hence a cusp at the kick. The amplitude and frequency of the wave are left to the fit. The phases of the contributing pues are taken to just be the geometric distance from the kick - in units of ags main magnets, and the pue amplitudes are not modified (as if the beta functions at the pues were all equal). We do not have better tune measurements - the tune meter was not yet functioning - so will use an average of these to get beta functions. The variation in the fitted tunes there is only one tune - give an indication of the associated error in that parameter. The sixth and seventh give the horizontal orbit response at the activated dipole (there is a clear cusp at the dipole - as there must be). In AGS, the corrector dipoles are physically built around the PUE cans. This position (column 6) is proportional to the beta function at the kick. More about this in the next paragraph.

The orbit shift at an excited dipole quite generally - assuming a linear response - is proportional to the beta function at that dipole, $\mathrm{x}=$ $(1 / 2)(\mathrm{kick}){ }^{*}$ beta*$^{*} \cot \left(\mathrm{Q}^{*} \mathrm{pi}\right)$. We learn the orbit shift from the average orbit along with an error from the standard deviation for that orbit. For the \#8 horizontal kick shown in figure 1 , the shift result is $4.24 \mathrm{~mm}+/-0.4 \mathrm{~mm}$. The kick strength (from the applied currents of $+/-4 \mathrm{~A}$, the magnet transfer function of $2.827 \mathrm{e}-4 \mathrm{Tm} / \mathrm{A}$, and the beam rigidity at injection $3.747 \mathrm{Tm}$ ) is 0.604 mradians. As has been described above, we extract the necessary tune from the data using a slightly more complicated model. From this fitting, a value for the tune results which is $(8.735(2)$ , 8.755(1)) for (hori, vert). This is the average for the average orbits from the four dipole locations so the set is at least self consistent. The extracted horizontal beta function at a \#8 straight section is then $15.5 \mathrm{~m}$ with an error of $+/-1 \mathrm{~m}$ driven more by the errors in position than in tune.

Turning to the response in the other plane, we see very systematic behavior and are led to make the same assumption of superperiodicity for whatever is 
responsible for this coupled motion. The result for the kicks at the horizontal \#2 straight section is given in figure 2 . The black dots are the average response at a given phase from the kick. The error bars are the standard deviation of the response for kicking with the dipole in the \#2 straight section but in different superperiods - same dance as with the in-plane response. The superperiod to superperiod variation is not larger than in the in-plane data. In the particular set on figure 2 , the average sigma is $.18 \mathrm{~mm}$ with a standard deviation over the ring of $0.06 \mathrm{~mm}$.

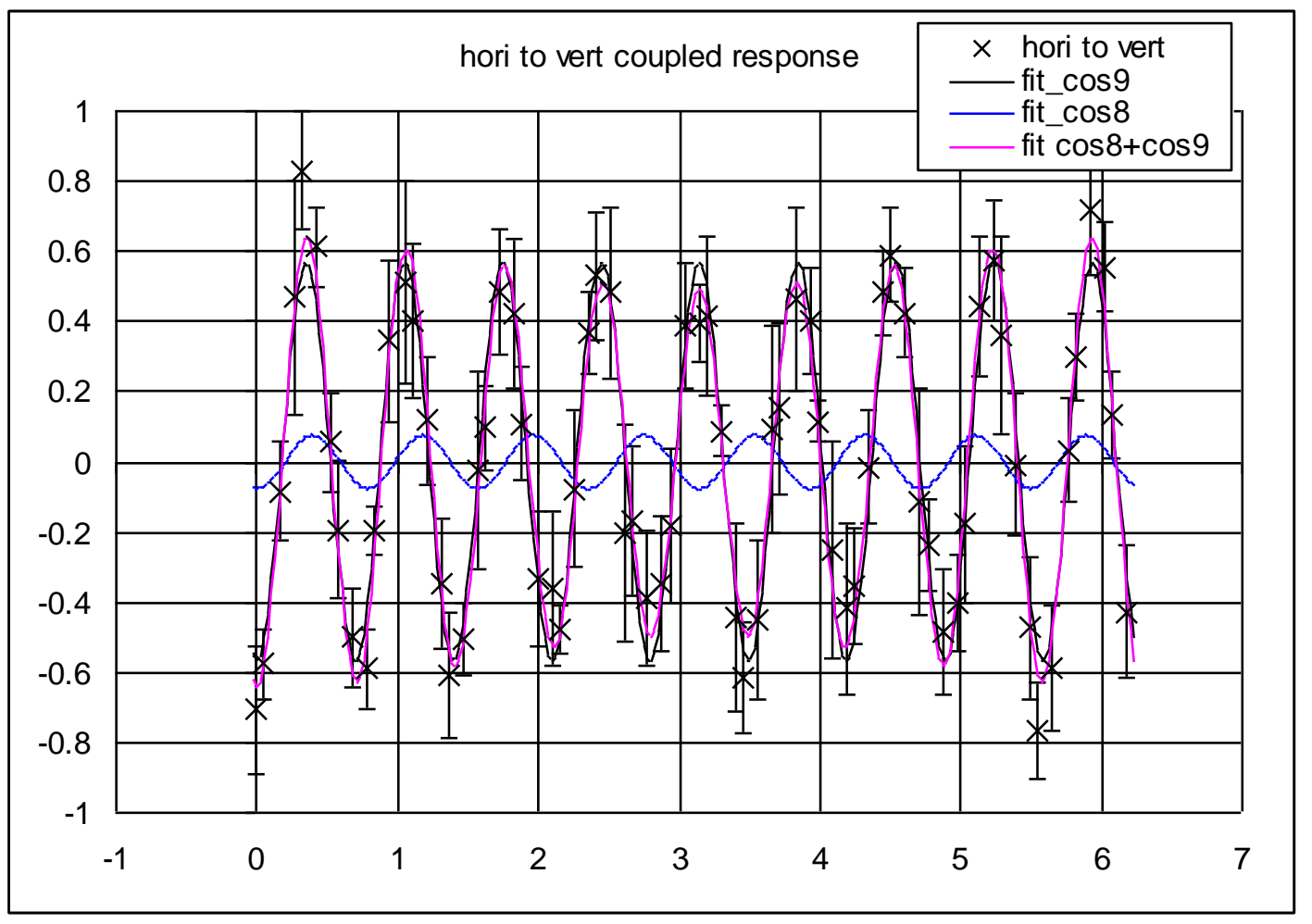

Figure 2 Average coupled response in the vertical plane from a kick at the \#2 horizontal dipoles.

To quantify this orbit, it is fit to 8th and 9th harmonics, with the phase zero reference set at the kick location, and the phase shift again just given by the number of main magnets away from the kick normalized to 2 pi for the full turn. The fitting wants almost pure cosine, mostly 9th but with some significant 8 th. That this same response applies well to kicks at the same correctors in different superperiods would be explained if whatever is responsible for the coupling is superperiodic. This would be sufficient, but is perhaps not necessary. This apparent coupling superperiodicity observation, made by J.W. Glenn (polarized proton meeting July 2009?) would already be very constraining and hence very useful. Historically we have ascribed the well known AGS coupling to randomly located rolled main magnets - magnets that have undergone some trauma - and to sagging magnets. The sagging hypothesis is just fine with a superperiodic effect of course. 
Above the results from the horizontal kicking at a \#2 was shown. If the same analysis is applied to the sets of kicks at the other dipole locations (i.e. \#8, \#12, \#18) the same conclusion follows. The responses all overlay when plotted relative to the kick. Table 2 gives the fitted 8th and 9th harmonics.

\begin{tabular}{|c|c|c|c|c|c|c|}
\hline \multirow{2}{*}{$\begin{array}{l}\text { powered } \\
\text { dipole \# }\end{array}$} & \multicolumn{4}{|c|}{ vert response } & \multirow{2}{*}{$\begin{array}{l}\text { PUE } \\
\text { sigma }\end{array}$} & \multirow{2}{*}{$\begin{array}{l}\text { PUE } \\
\text { sd }\end{array}$} \\
\hline & $\sin 9$ & $\cos 9$ & $\sin 8$ & $\cos 8$ & & \\
\hline 2 & 0.015 & -0.567 & -0.013 & -0.076 & 0.18 & 0.06 \\
\hline 8 & -0.052 & -0.562 & -0.002 & -0.077 & 0.19 & 0.06 \\
\hline 12 & -0.036 & -0.531 & 0.001 & -0.079 & 0.19 & 0.06 \\
\hline 18 & -0.018 & -0.524 & -0.010 & -0.080 & 0.17 & 0.04 \\
\hline
\end{tabular}

Table 2 Vertical harmonics in the coupled response to horizontal dipoles at the listed straight sections, injection, au

Inspection of the table, and of the data, shown in Appendix II , reveals the further result that here as in the horizontal "in plane" response, there is little change in the relative response as one moves from one dipole location to the next. So the cause for the coupling is consistent with an effect that is not only superperiodic but indeed has the approximate 60 -fold periodicity of the AGS. The coupled response is consistent with what would occur if there were vertical dipoles distributed uniformly around the ring and powered proportional to the horizontal offset. To get the sign of the required fields right is as usual problematic. However we are convinced that 1 ) the signs of the pues have the definition that + means up or beam right (= "out" in radius) and 2) the dipoles follow the Bleser convention so positive current means a bend up or beam left.

Up to now the data resulting from horizontal dipole excitation at gold injection has been discussed. Now the same analysis using vertical dipole data is examined, and again at gold injection. The results shown in appendix III and IV are very similar to those from the horizontal. Summary tables for the vertical dipoles, identical in form to those show above for the horizontal are given in tables 3 and 4 .

\begin{tabular}{|c|c|c|c|c|c|c|}
\hline \multirow{2}{*}{$\begin{array}{l}\text { powered } \\
\text { dipole \# }\end{array}$} & \multicolumn{2}{|c|}{ superperiodicity } & \multicolumn{2}{|l|}{$\begin{array}{l}\text { simple } \\
\text { fit }\end{array}$} & \multicolumn{2}{|c|}{$\begin{array}{l}\text { V pos at dipole } \\
\text { (ave) }\end{array}$} \\
\hline & $\mathrm{mm}$ & $\mathrm{mm}$ & $\mathrm{Mm}$ & & $\mathrm{mm}$ & sig \\
\hline 2 & 0.20 & 0.06 & 6.55 & 8.756 & -4.62 & 0.20 \\
\hline 8 & 0.21 & 0.08 & 6.58 & 8.754 & -4.61 & 0.33 \\
\hline 12 & 0.23 & 0.06 & 6.78 & 8.754 & -4.88 & 0.17 \\
\hline 18 & 0.21 & 0.07 & 6.58 & 8.756 & -4.74 & 0.28 \\
\hline
\end{tabular}

Table 3: vertical orbit response to vertical dipoles

The amplitude of the sinusoidal response in the excited plane, now vertical, column four, is about $6.5 \mathrm{~mm}$, slightly larger than for the horizontal dipoles, and 
very similar first among the orbits generated by the same superperiod position dipoles, but indeed also for the dipoles at all of the superperiod positions. These orbits are shown in appendix III. The rms variation for a pue at a given phase from the kick (column 2) is smaller here in the vertical than it was in the horizontal, 0.2 $\mathrm{mm}$ vs $0.3 \mathrm{~mm}$. 
in table 4.

The coupled response from these vertical dipoles is given in appendix IV and

\begin{tabular}{|c|c|c|c|c|c|c|}
\hline powered & $\begin{array}{l}\text { Hori } \\
\text { resp }\end{array}$ & & & & pue & \\
\hline dipole \# & $\sin 9$ & $\cos 9$ & $\sin 8$ & $\cos 8$ & sigma & sd \\
\hline 2 & -0.034 & 0.470 & 0.005 & 0.075 & 0.23 & 0.04 \\
\hline 8 & -0.021 & 0.464 & 0.000 & 0.080 & 0.20 & 0.05 \\
\hline 12 & -0.036 & 0.475 & -0.006 & 0.091 & 0.23 & 0.04 \\
\hline 18 & -0.001 & 0.426 & -0.003 & 0.094 & 0.22 & 0.32 \\
\hline
\end{tabular}

Table 4: horizontal response to vertical dipoles

The dominant harmonic is cosine ( 9 theta) where again the phase zero reference is defined to be at the kick. As before, the coupled response is nearly the same for all the dipole locations. The coupled "horizontal from vertical" is slightly weaker than the "vertical from horizontal" $(\sim 0.46 \mathrm{~mm}$ hori from $\sim 6.5 \mathrm{~mm}$ vertical $)$ vs ( $\sim 0.55 \mathrm{~mm}$ vertical from $\sim 6 \mathrm{~mm}$ horizontal). Taking these as fractions for further comparison then the horizontal primary motion couples over about .09 in amplitude and vertical about .07 .

\section{More discussion:}

Can we now propose an addition to the model that would give this coupling, and to what extend is that solution unique and extendable to other rigidities? For the vertical dipole excitation the vertical orbit distortion (though really an oscillation at the tune noninteger harmonic with a cusp at the kick to close despite the non-integer tune) is parameterized by a 9 th harmonic with a $6 \mathrm{~mm}$ amplitude and a peak opposite - 180 degrees around the ring - from the kick. The coupled horizontal is also primarily 9th with $-0.4 \mathrm{~mm}$ amplitude opposite the kick. For the horizontal dipoles, the similar orbit numbers are: horizontal $6 \mathrm{~mm}$ inward and resulting vertical $0.5 \mathrm{~mm}$ up. Such a response would result if a uniformly distributed skew quad field were added around the ring. Since the tunes are below 9, in both planes the 9th harmonic orbit distortion is opposite in sign to the driving 9 th harmonic dipole field. So what we need in the vertical plane is a magnetic field that points away from the origin and grows with the magnitude of the vertical displacement and in the horizontal a field pointing toward the origin that grows with the magnitude of the horizontal displacement. By the Bleser convention, we need a "skew" quad with positive current. We would get the right effect if we rotate the focusing ags main magnets clockwise along the beam direction and the defocusing magnets counterclockwise. (This reasoning is open to sign challenges).

How many little coupled kicks would it take to make the associated cusps be lost in the orbit noise? Without cusps, the only features of the response are the amplitudes and phases of the $9^{\text {th }}$ and $8^{\text {th }}$. The phase is apparently set to be opposite the kick - just like the in-plane response. To what extent does the observation that 
kicks in different superperiods give the same response require superperiodic skew terms as opposed to just many randomly distributed skew terms?

\section{Results from high rigidity:}

Such a model change would most simply remain the same in geometry as the main magnet field in the AGS increases. Then we would expect to see the same coupling for the bare AGS at high field. We turn to the data taken with the gold accelerated to RHIC injection energy - AGS rigidity up by x20 but kick strength up by only x 5 (power supply limits) so amplitude of in-plane motion down by about 4 . First: two representative pictures for vertical orbit distorting: figure 3 gives the direct response, figure 4 the coupled response. In this case the fitting to the coupled response is not particularly convincing - whether the extracted numbers carry any truth about the coupling - surely a limit on the size however.

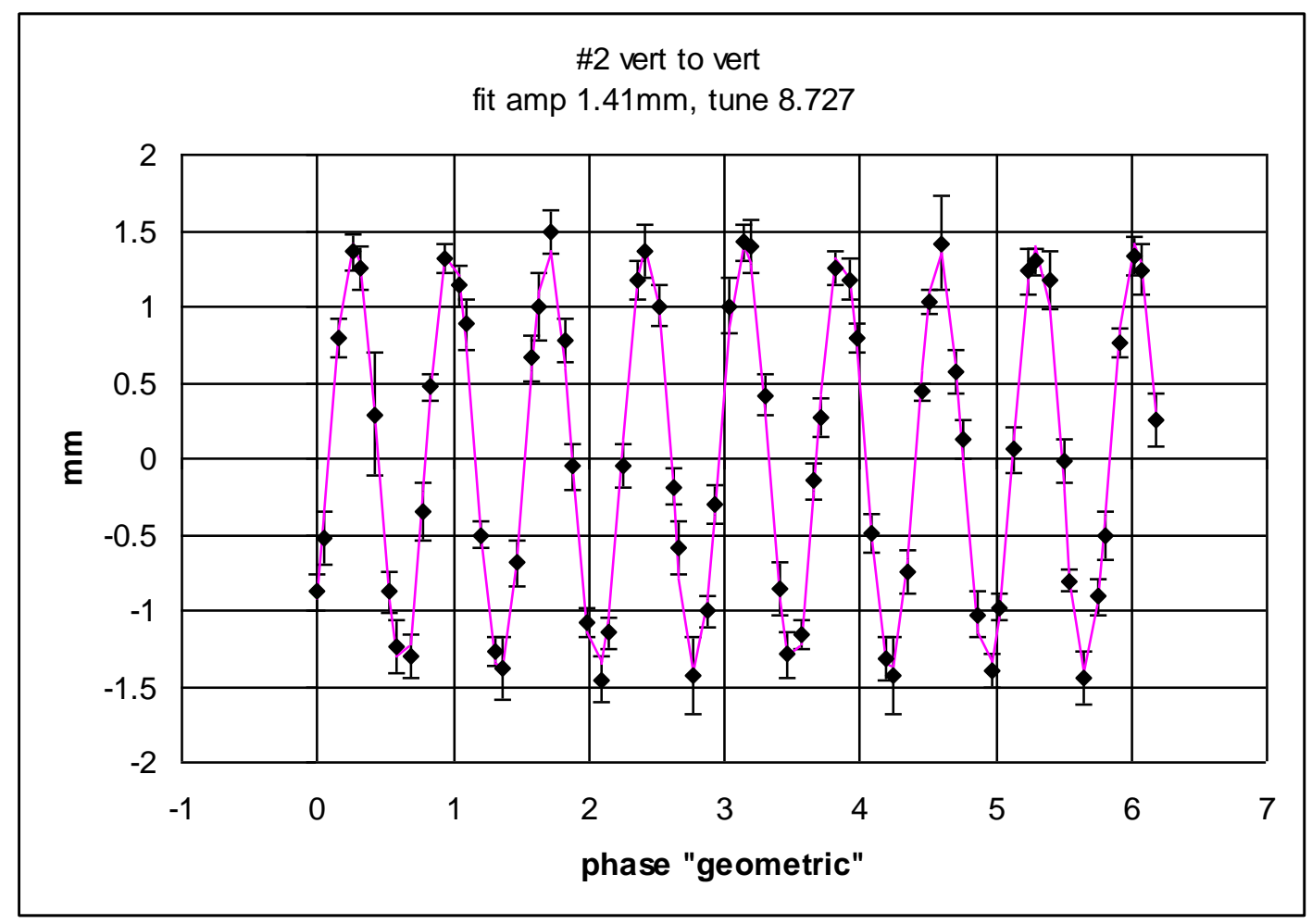

Figure 3 Vertical orbit response to adding a vertical dipole 


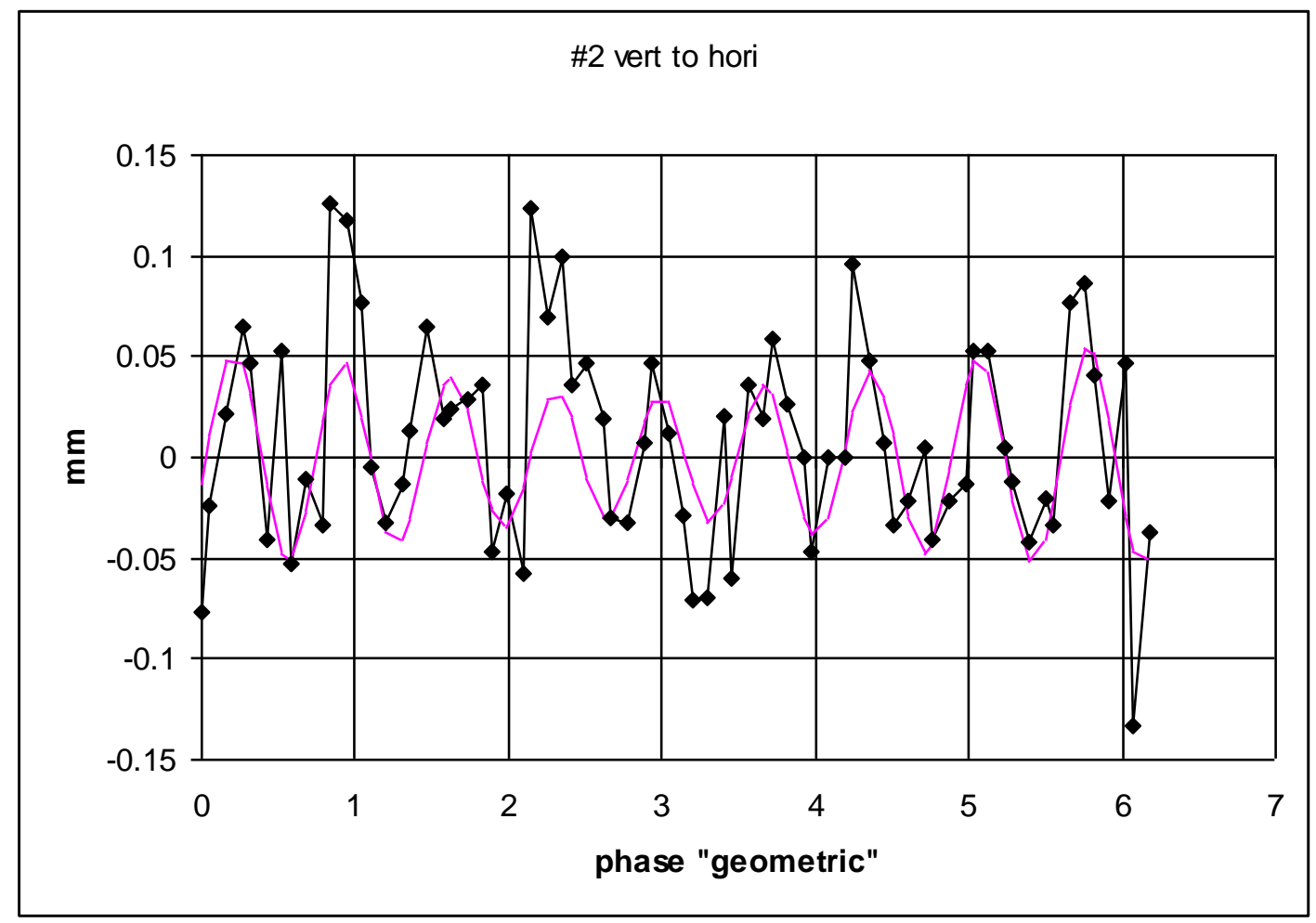

Figure 4 The coupled response in the horizontal plane from the vertical dipole

The extracted numbers from the four dipole positions for the vertical dipoles are given in table 5 and 6 . As expected the amplitude of the in-plane response is down from $6 \mathrm{~mm}$ to $1.5 \mathrm{~mm}$, the factor of four. The variation in position for a given phase from the kick is slightly smaller ( 0.15 vs 0.2$)$ than the injection data.

\begin{tabular}{|c|c|c|c|c|c|c|}
\hline $\begin{array}{l}\text { v resp } \\
\text { v kick }\end{array}$ & $\begin{array}{l}\text { superperiodici } \\
\text { sigma pue } \\
\mathrm{Mm}\end{array}$ & $\begin{array}{l}\mathrm{y} \\
\mathrm{sd} \\
\mathrm{mm}\end{array}$ & $\begin{array}{l}\text { simple } \\
\text { fit } \\
\text { Amp } \\
\mathrm{mm}\end{array}$ & tune & $\begin{array}{l}\text { Vpos at kick } \\
\text { "sp ave" } \\
\text { mm }\end{array}$ & $\begin{array}{l}\text { sigma } \\
\mathrm{mm}\end{array}$ \\
\hline 2 & 0.14 & 0.05 & 1.41 & 8.727 & -0.88 & 0.12 \\
\hline 8 & 0.15 & 0.05 & 1.41 & 8.719 & -0.80 & 0.20 \\
\hline 12 & 0.14 & 0.03 & 1.38 & 8.727 & -0.91 & 0.12 \\
\hline 18 & 0.15 & 0.04 & 1.33 & 8.725 & -0.84 & 0.18 \\
\hline
\end{tabular}

Table 5 In-plane response summary. Column 1: kick location, column 2: standard deviation of reported position at a given phase relative to the kick and variation of this sigma , column 3: amplitude and tune for "simple" fitting, 4: average motion at the dipole - for extraction of beta function there. 


\begin{tabular}{|c|c|c|c|c|c|}
\hline $\sin 9$ & $\cos 9$ & $\sin 8$ & $\cos 8$ & $\begin{array}{l}\text { sigma } \\
\text { pue }\end{array}$ & $\mathrm{Sd}$ \\
\hline 0.042 & -0.008 & 0.010 & -0.006 & 0.13 & 0.03 \\
\hline 0.018 & 0.003 & 0.006 & 0.001 & 0.12 & 0.04 \\
\hline 0.015 & 0.007 & 0.021 & -0.002 & 0.12 & 0.03 \\
\hline 0.016 & -0.006 & 0.014 & -0.004 & 0.13 & 0.04 \\
\hline
\end{tabular}

table 6 Response in the not kicked plane.

The coupled motion figure does not include the sigmas - which are larger ( 0.13 in the above table) than any structure. One would argue that this overestimates the average error by the factor $\sqrt{ }(1 / \mathrm{n})$ which would be about $1 / 3$, but this is still the size of the observed effect. There is some consistency across the four kicks with the sin 9 theta being always the largest component.

One could turn this around and ask what would be expected if the rule at injection held here. The $\cos 9$ should be dominant, and the magnitude should be down by the factor four, so the $\cos 9$ amplitude would be a bit more than $0.1 \mathrm{~mm}$. This is inconsistent - much larger - than the numbers from the fits. and 8 .

Ok, and finally we show the high rigidity horizontal kicks results in tables 7

\begin{tabular}{|c|c|c|c|c|c|c|}
\hline h resp & superperiodici & & $\begin{array}{l}\text { Simple } \\
\text { fit }\end{array}$ & & Hpos at kich & \\
\hline h kick & $\begin{array}{l}\text { sigma pue } \\
\mathrm{mm}\end{array}$ & $\begin{array}{l}\mathrm{sd} \\
\mathrm{mm}\end{array}$ & $\begin{array}{l}\text { Amp } \\
\mathrm{Mm}\end{array}$ & tune & $\begin{array}{l}\text { "sp ave" } \\
\mathrm{mm}\end{array}$ & $\begin{array}{l}\text { sigma } \\
\mathrm{mm}\end{array}$ \\
\hline 2 & 0.16 & 0.04 & 1.35 & 8.717 & 0.88 & 0.17 \\
\hline 8 & 0.17 & 0.06 & 1.33 & 8.719 & 0.87 & 0.18 \\
\hline 12 & 0.16 & 0.04 & 1.33 & 8.721 & 0.86 & 0.21 \\
\hline 18 & 0.16 & 0.04 & 1.37 & 8.719 & 0.96 & 0.18 \\
\hline
\end{tabular}

Table 7 hori response in the kicked plane, gold extraction . Reasonable amplitudes and tunes and sigmas.

\begin{tabular}{|c|c|c|c|c|c|c|}
\hline \multirow{3}{*}{$\begin{array}{l}\text { v resp } \\
\text { h kick }\end{array}$} & & & & & \multicolumn{2}{|c|}{ superperiodicity } \\
\hline & vert resp & nse & & & sigma pue & sd \\
\hline & $\sin 9$ & $\cos 9$ & $\sin 8$ & $\cos 8$ & $\mathrm{Mm}$ & $\mathrm{mm}$ \\
\hline 2 & 0.001 & -0.047 & 0.017 & -0.025 & 0.14 & 0.06 \\
\hline 8 & -0.010 & -0.037 & -0.013 & -0.027 & 0.17 & 0.06 \\
\hline 12 & -0.012 & -0.029 & -0.005 & -0.009 & 0.16 & 0.05 \\
\hline 18 & 0.017 & -0.022 & 0.006 & -0.008 & 0.15 & 0.04 \\
\hline
\end{tabular}

Table $8 \mathrm{~h}$ to $\mathrm{v}$ coupled response, gold extraction. Here the $\cos 9$ is largest, but not a the expected $0.1 \mathrm{~mm}$ amplitude level. 
Appendix I : Horizontal orbit responses to horizontal dipole, gold injection

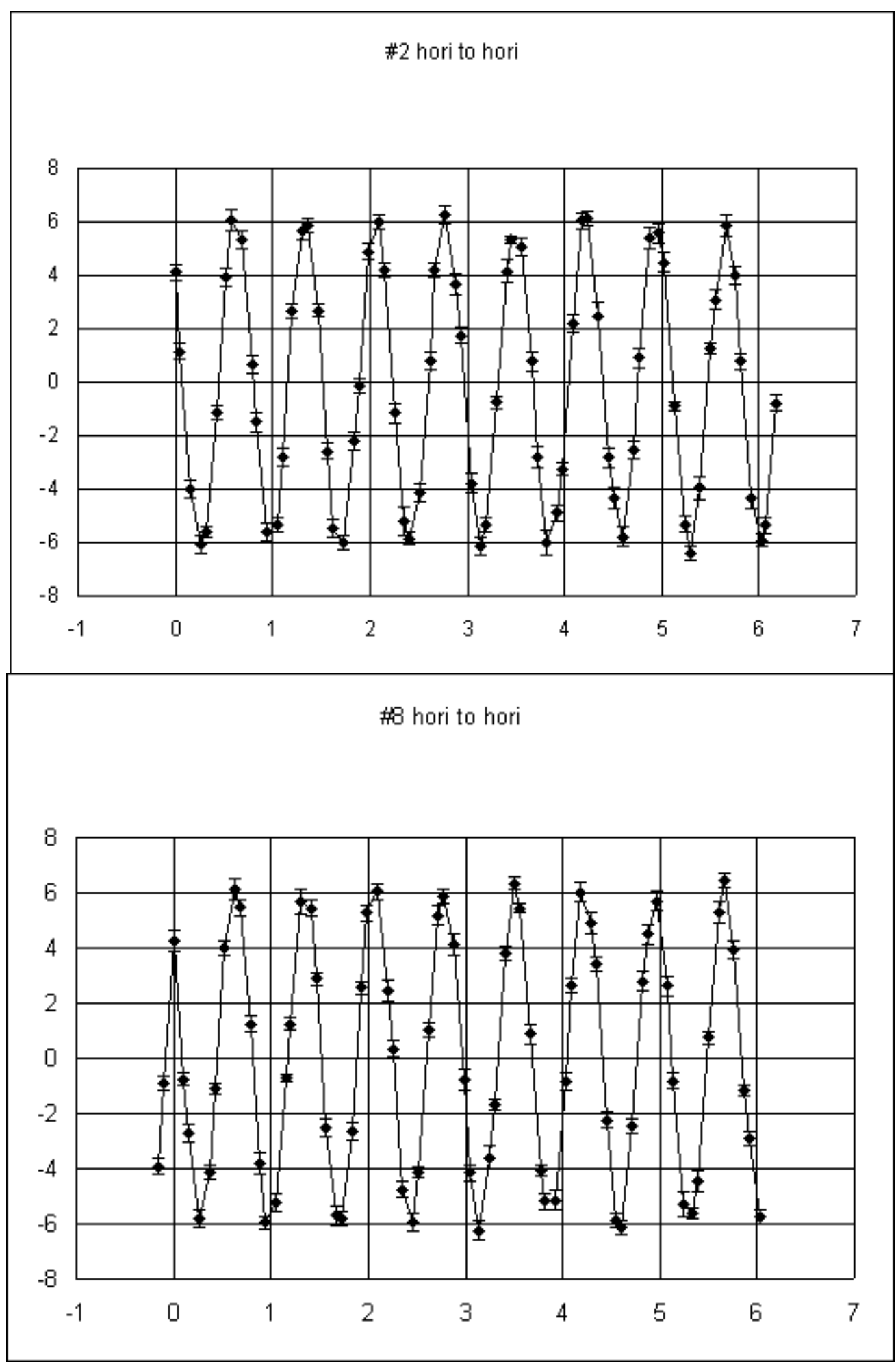




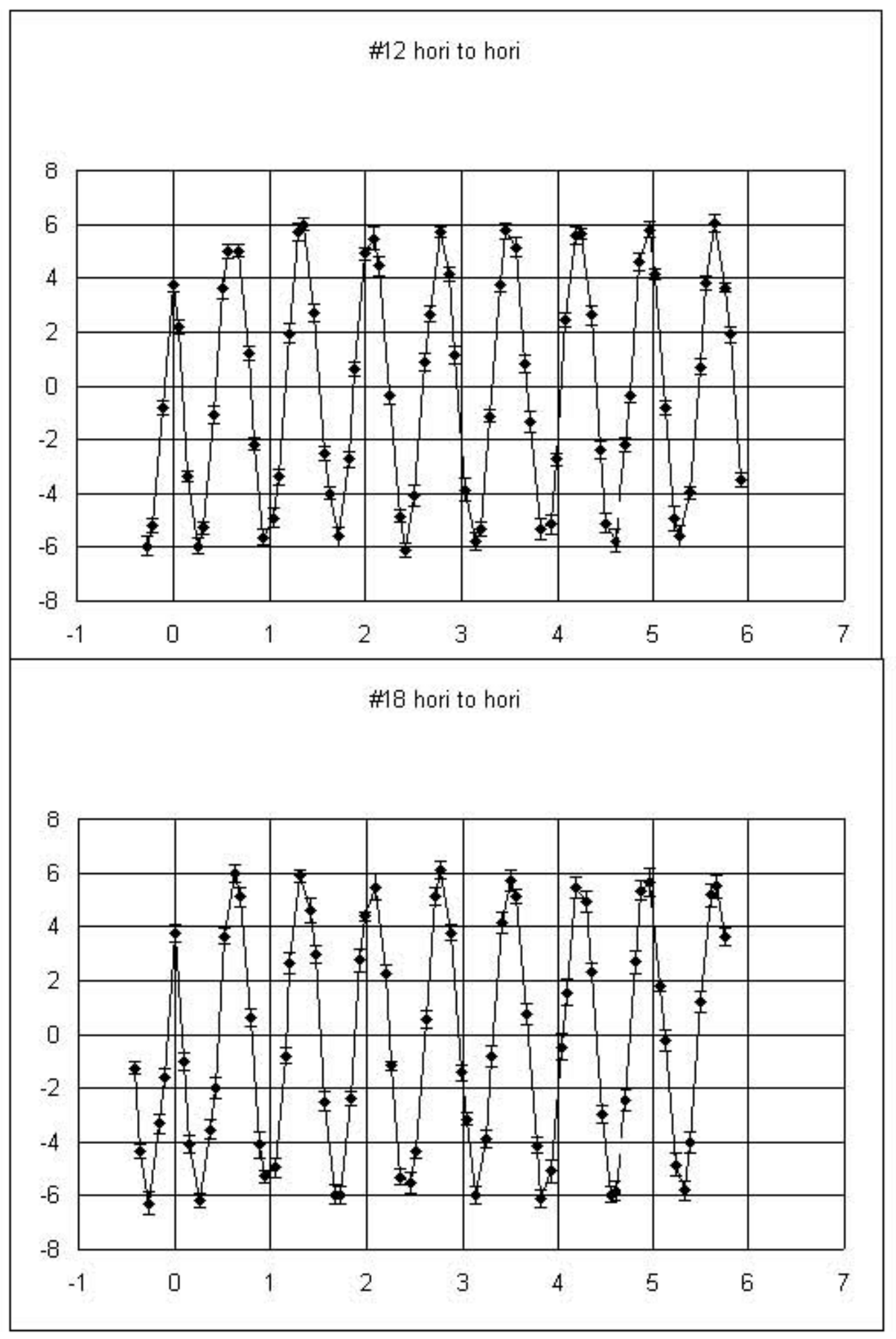


Appendix II: Vertical orbit response to horizontal dipoles, gold injection. Curve is the fit to 8th and 9th harmonics.

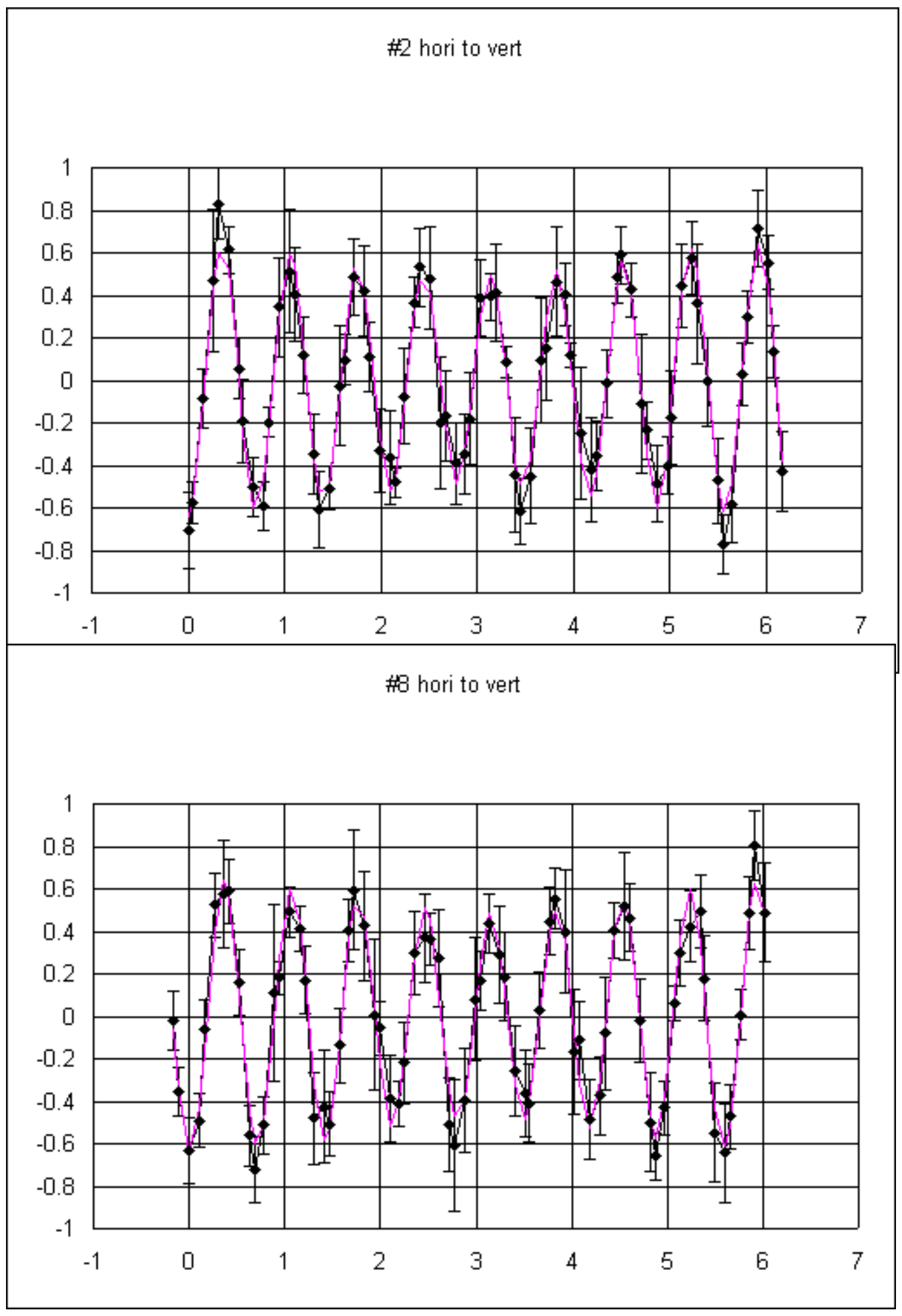




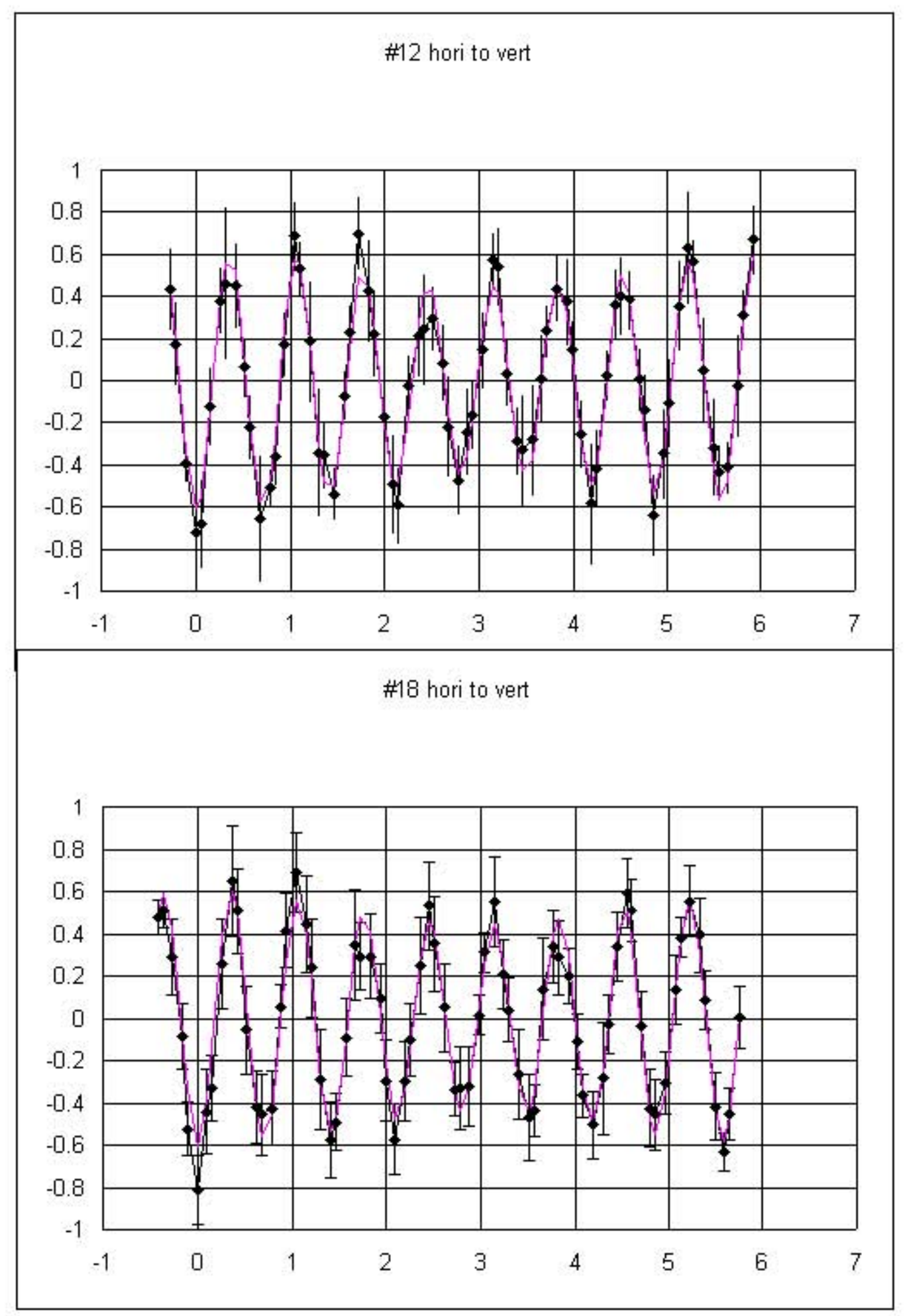


Appendix III: Vertical orbit responses to vertical dipoles, gold injection

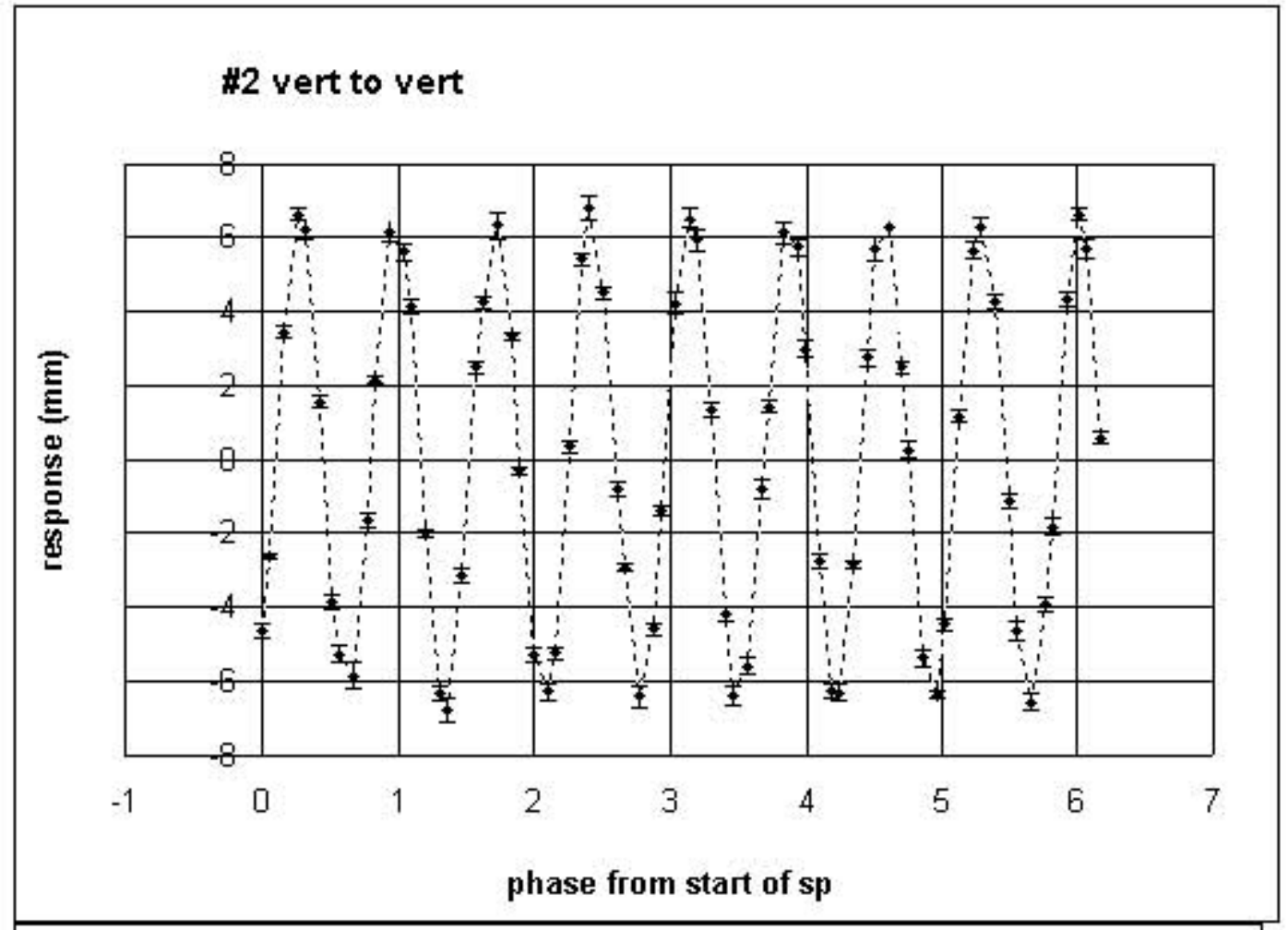

\#8 vert from vert

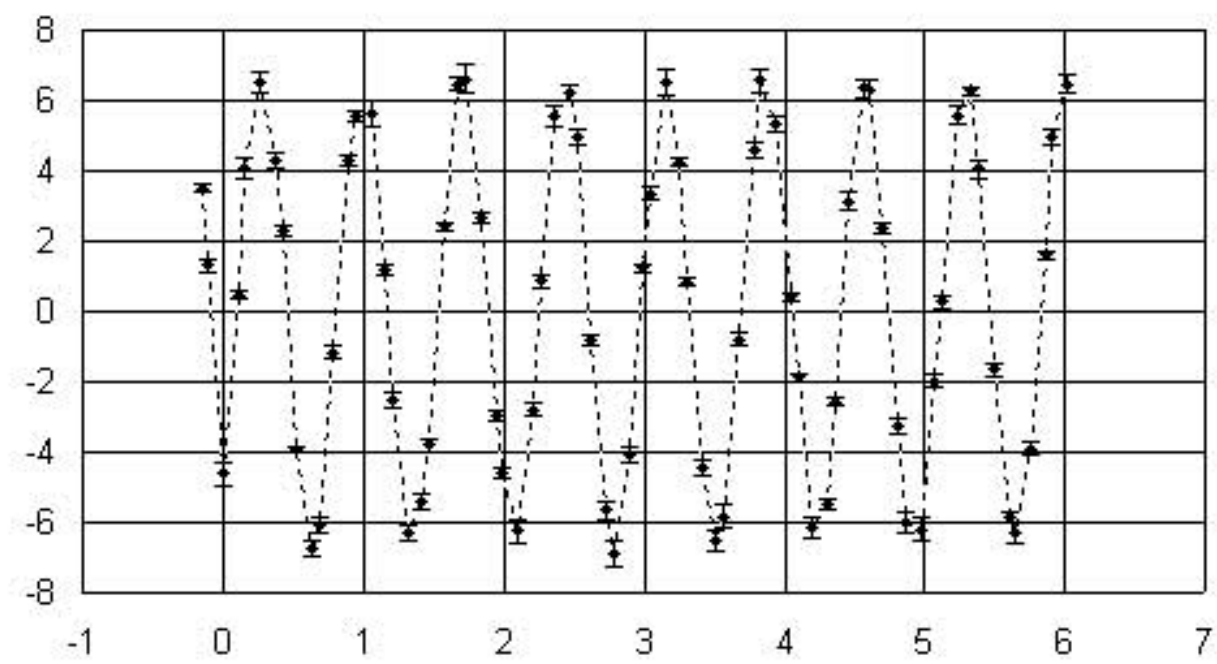




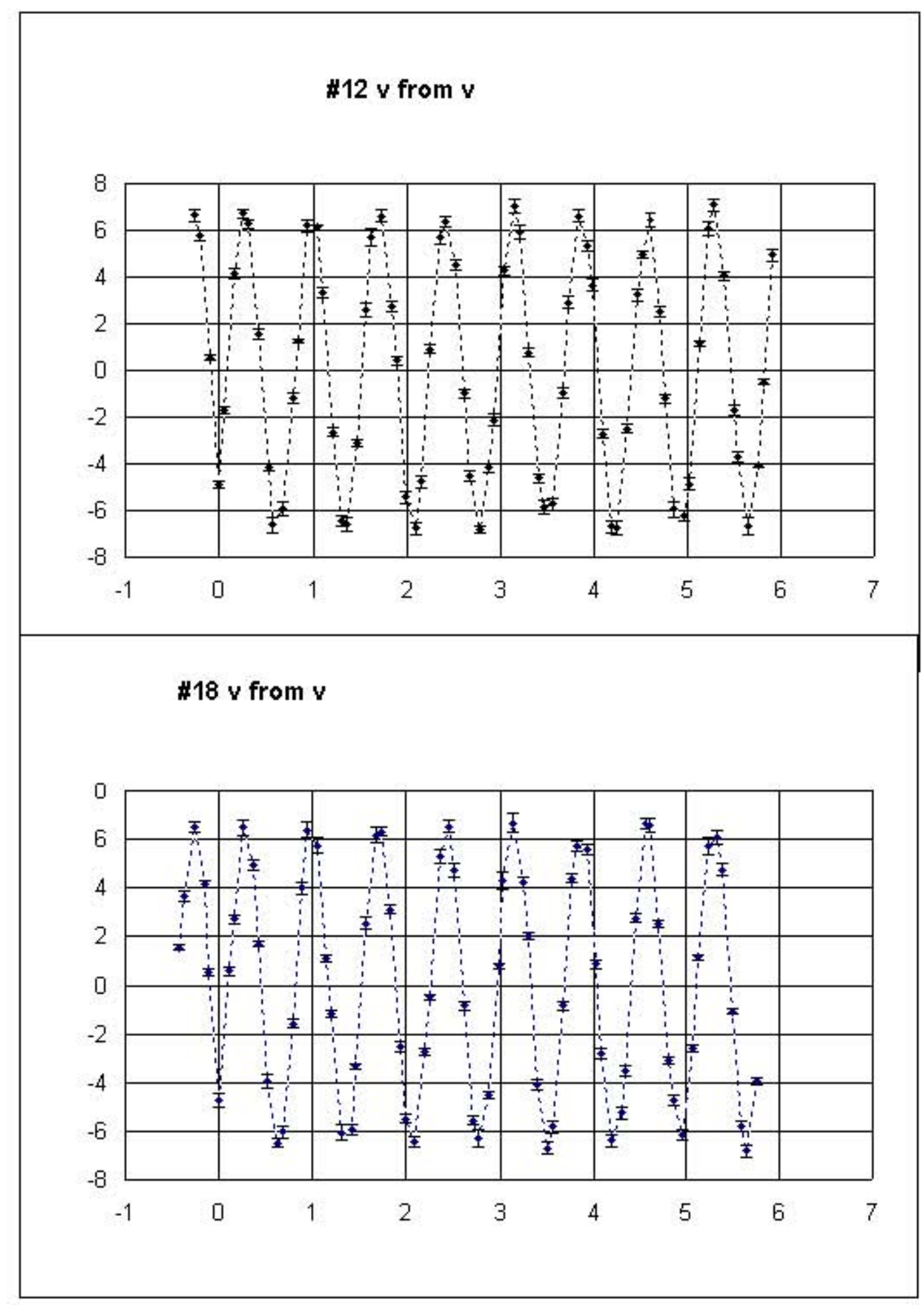


Appendix IV: Horizontal orbit response to vertical dipoles, gold injection. Curve is the fit to 8th and 9th harmonics.
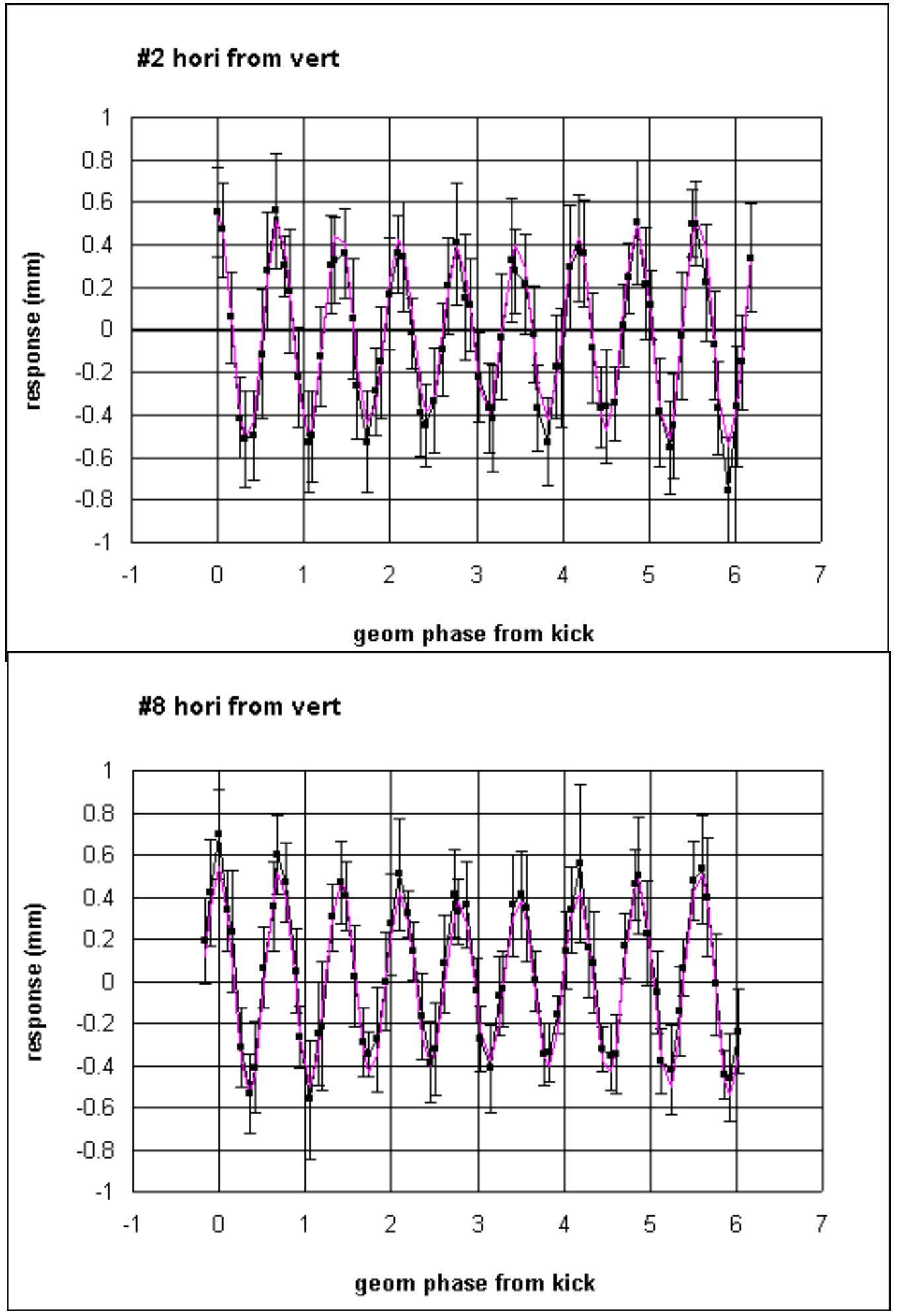


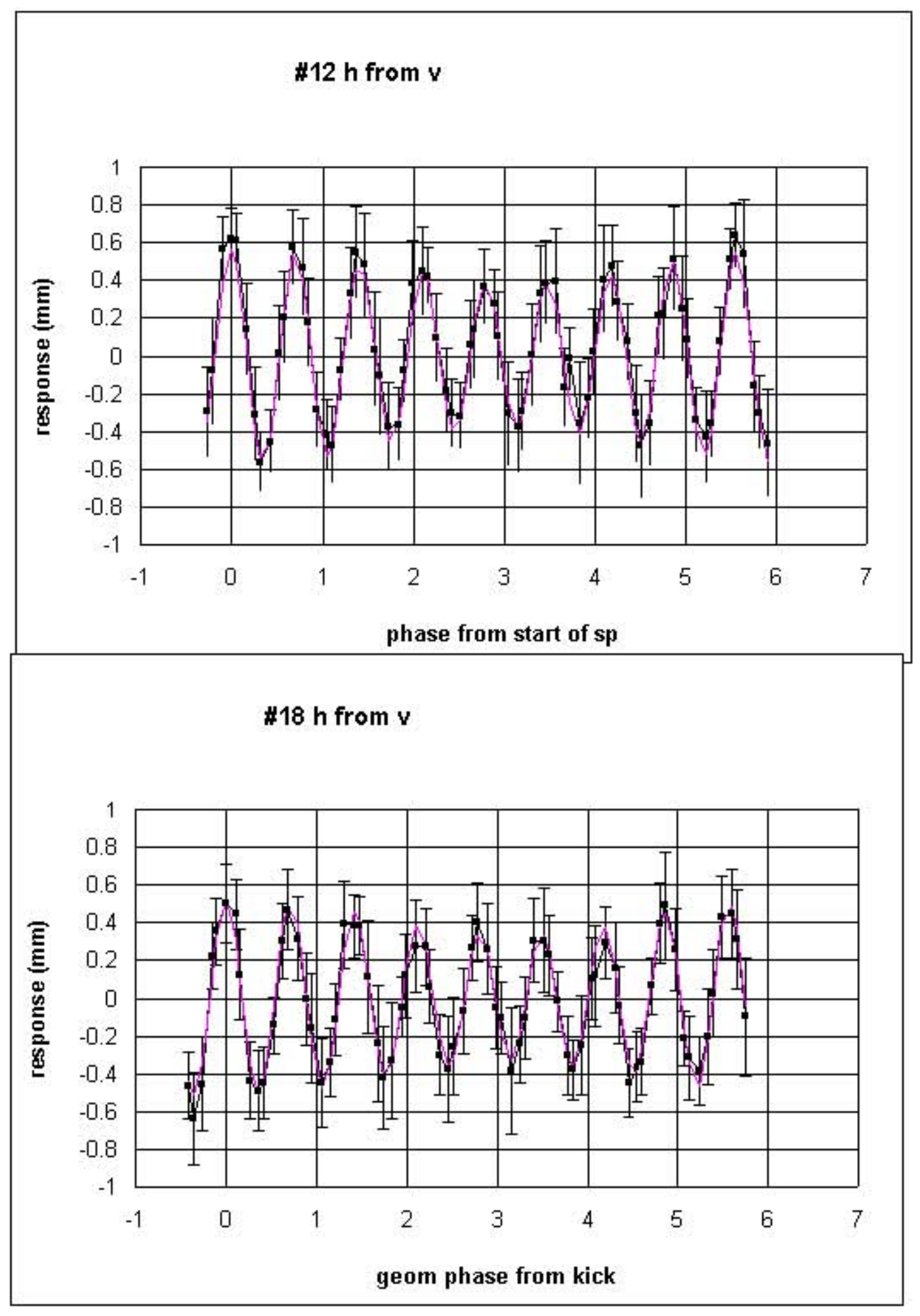


Appendix V: Horizontal orbit responses to horizontal dipoles, gold extraction

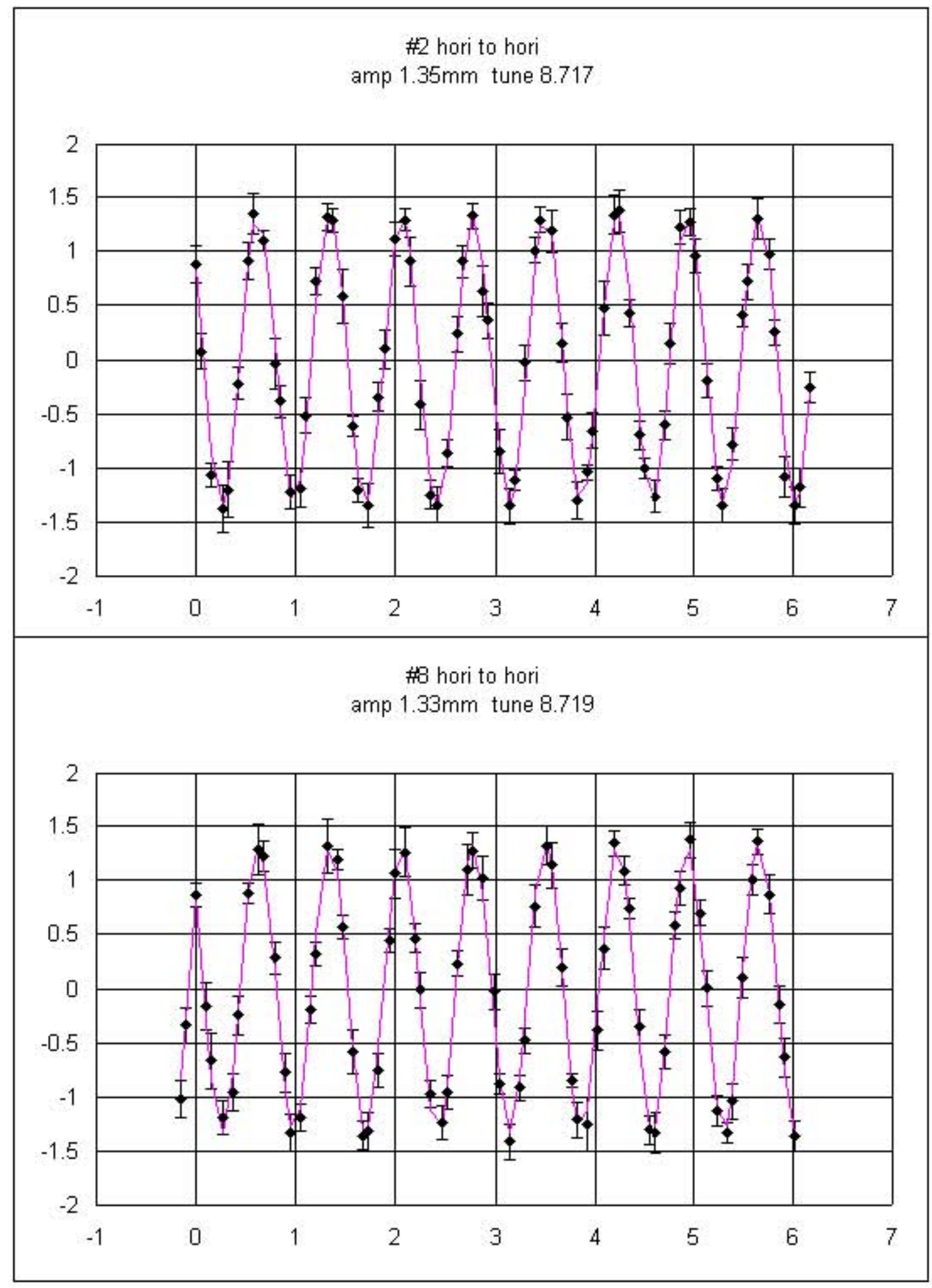




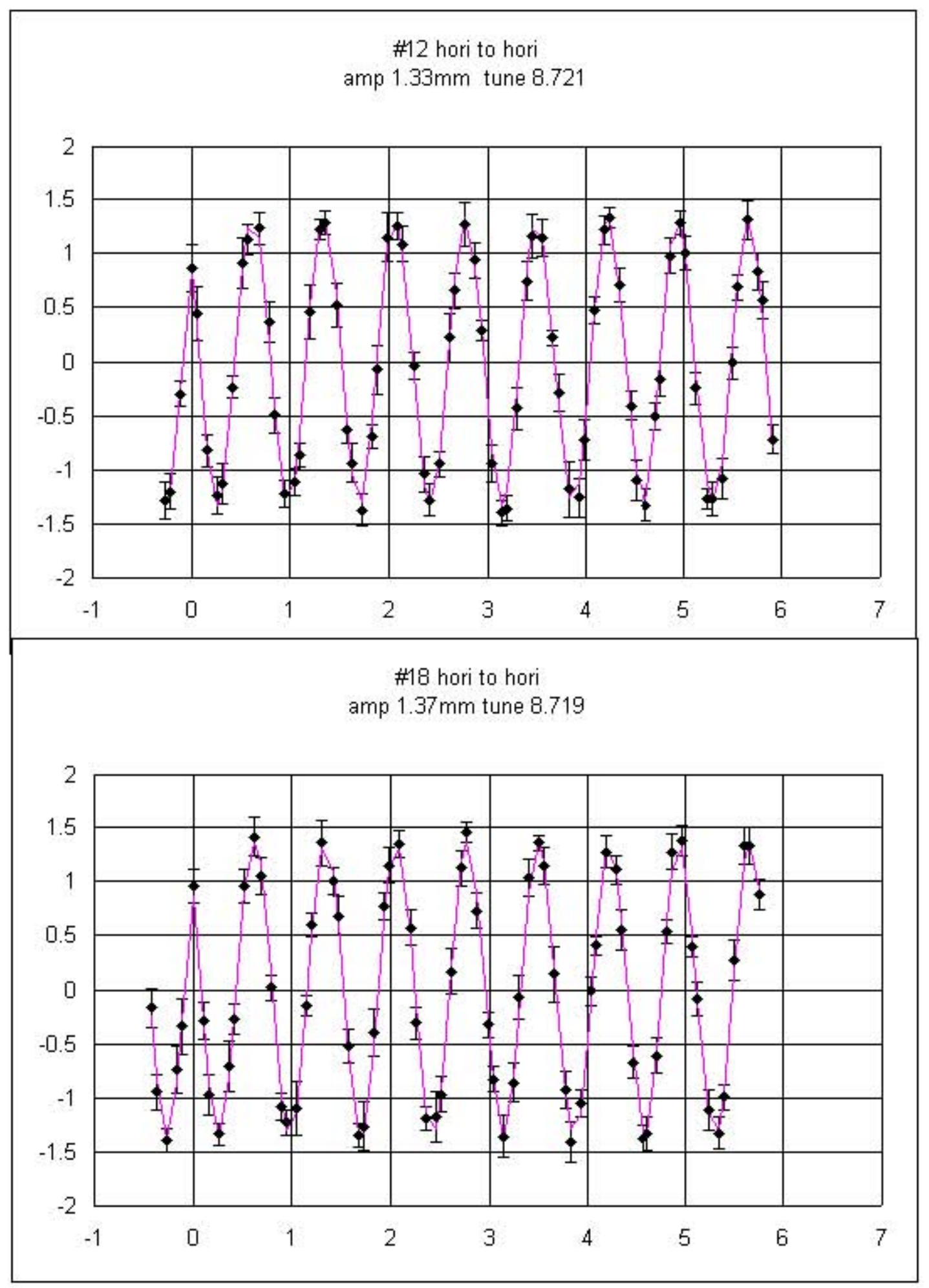


Appendix VI: Vertical orbit responses to horizontal dipoles, gold extraction

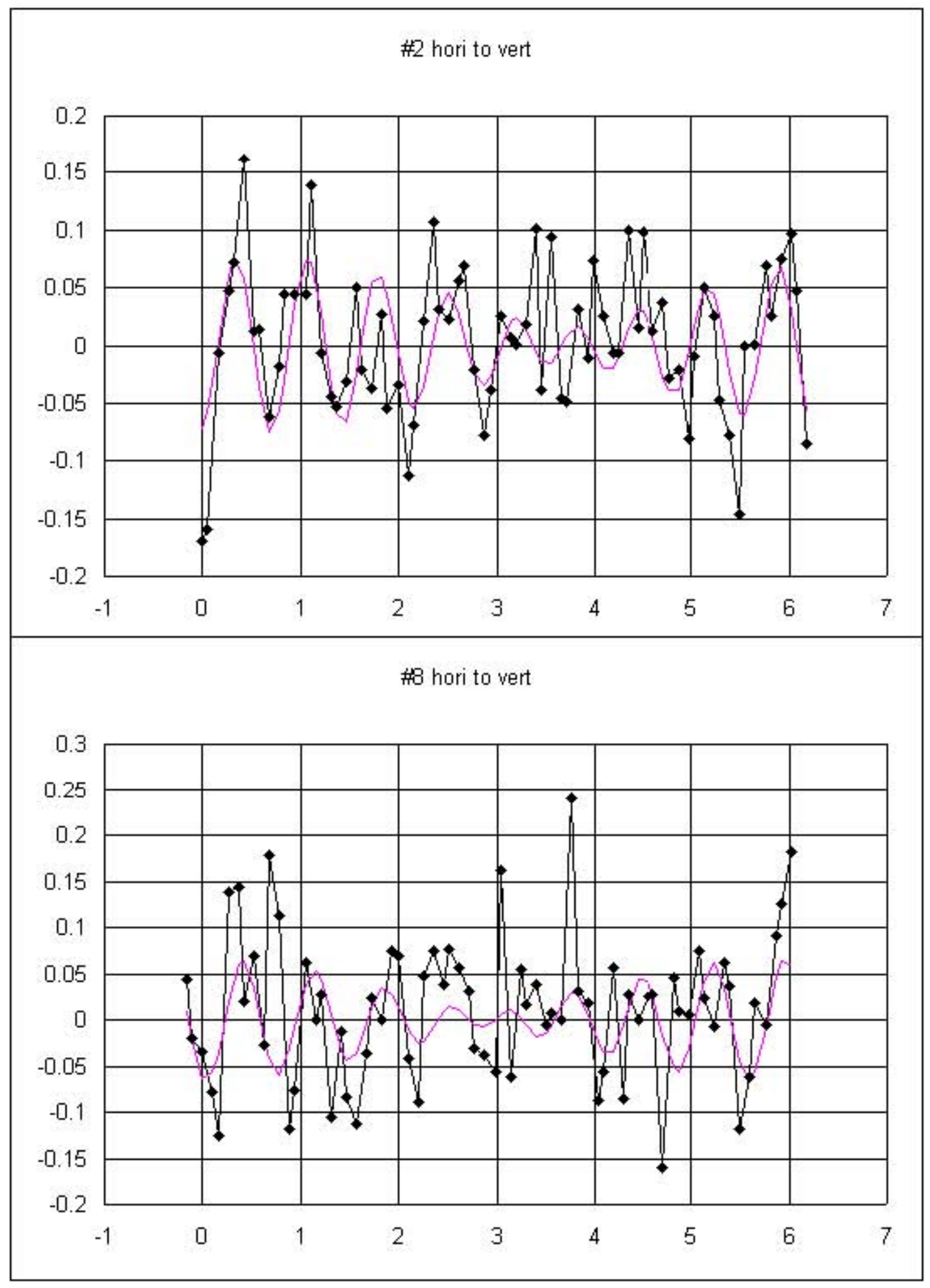




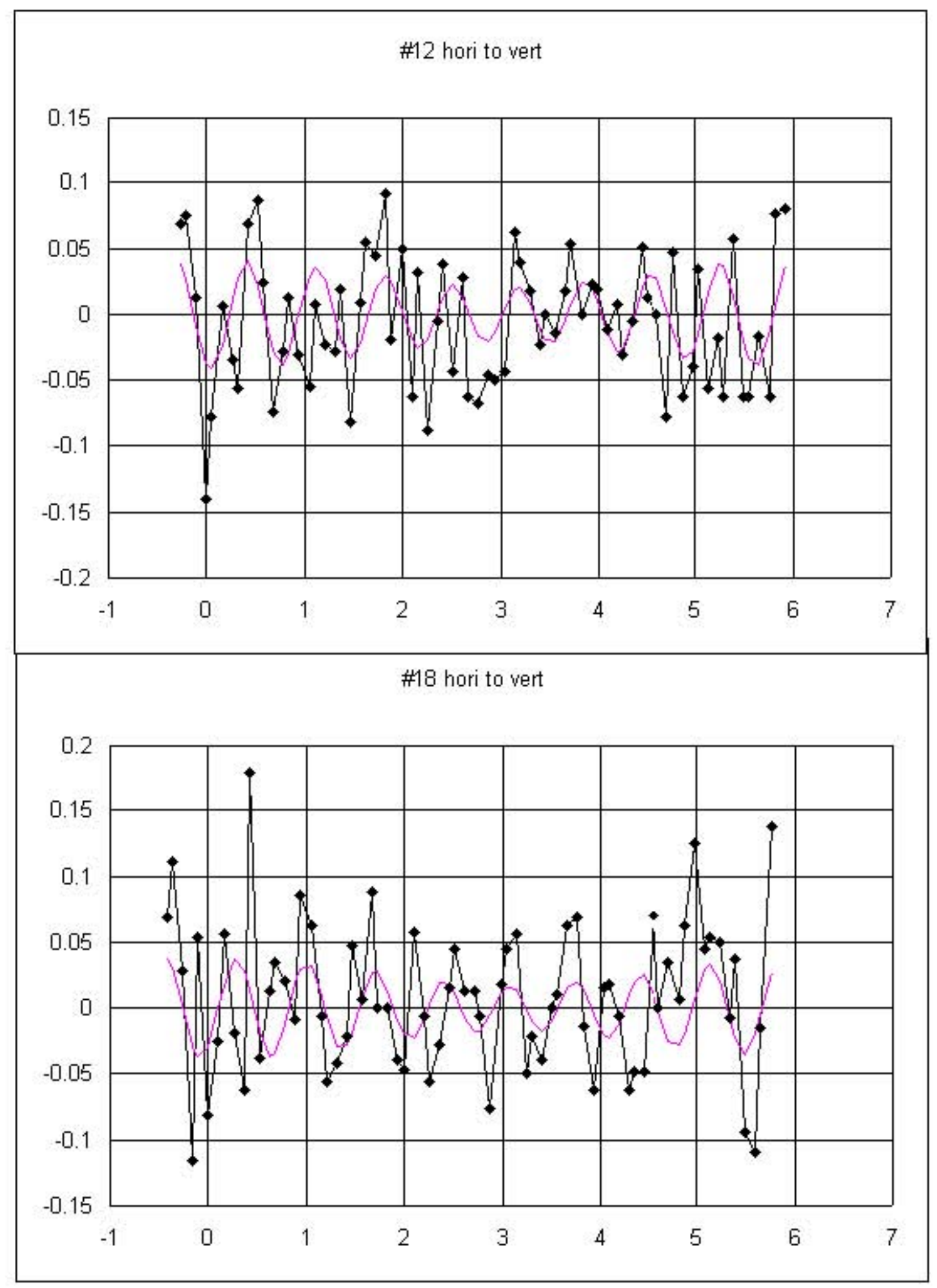


Appendix VII: Vertical orbit responses to vertical dipoles, gold extraction

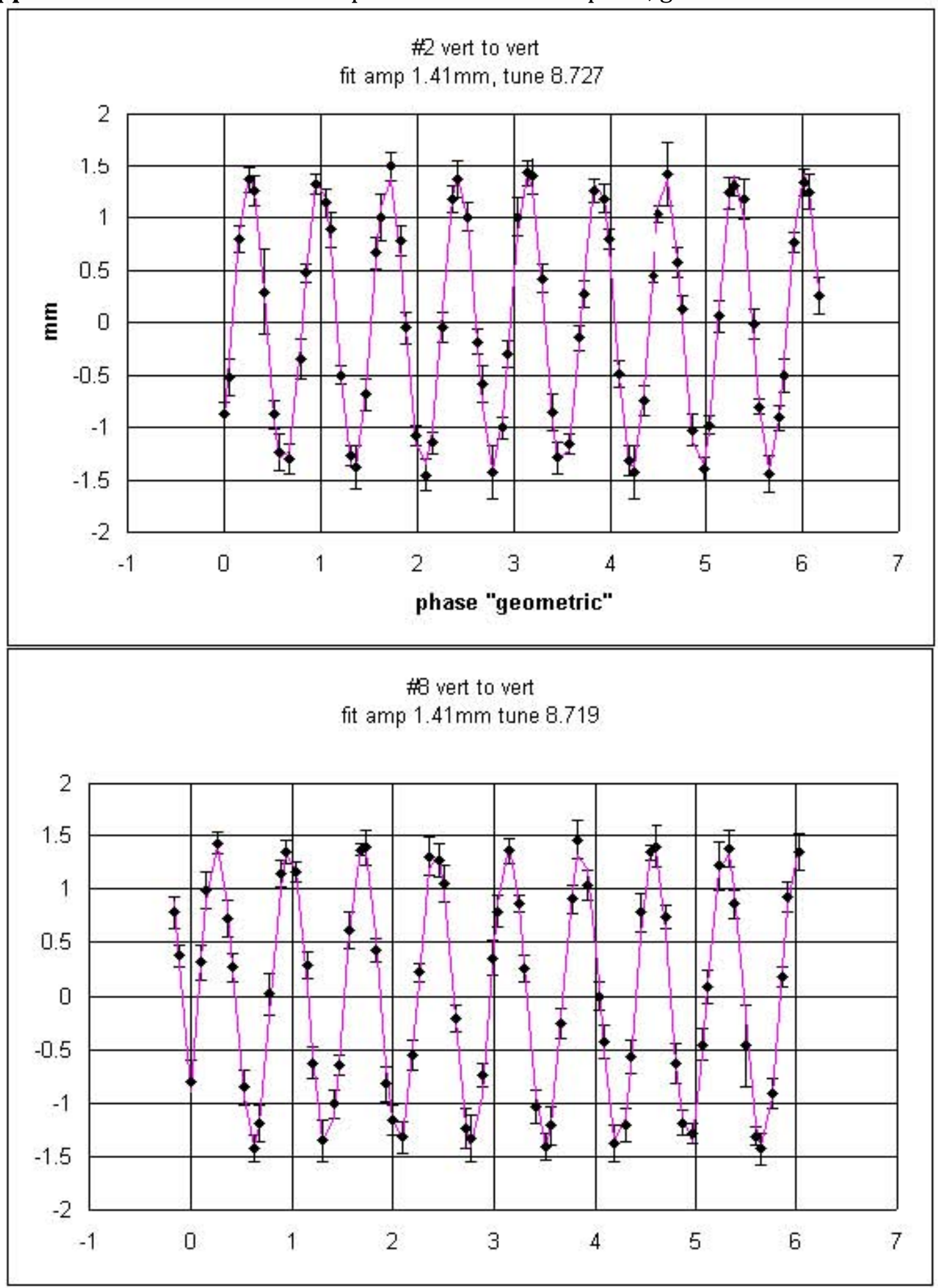




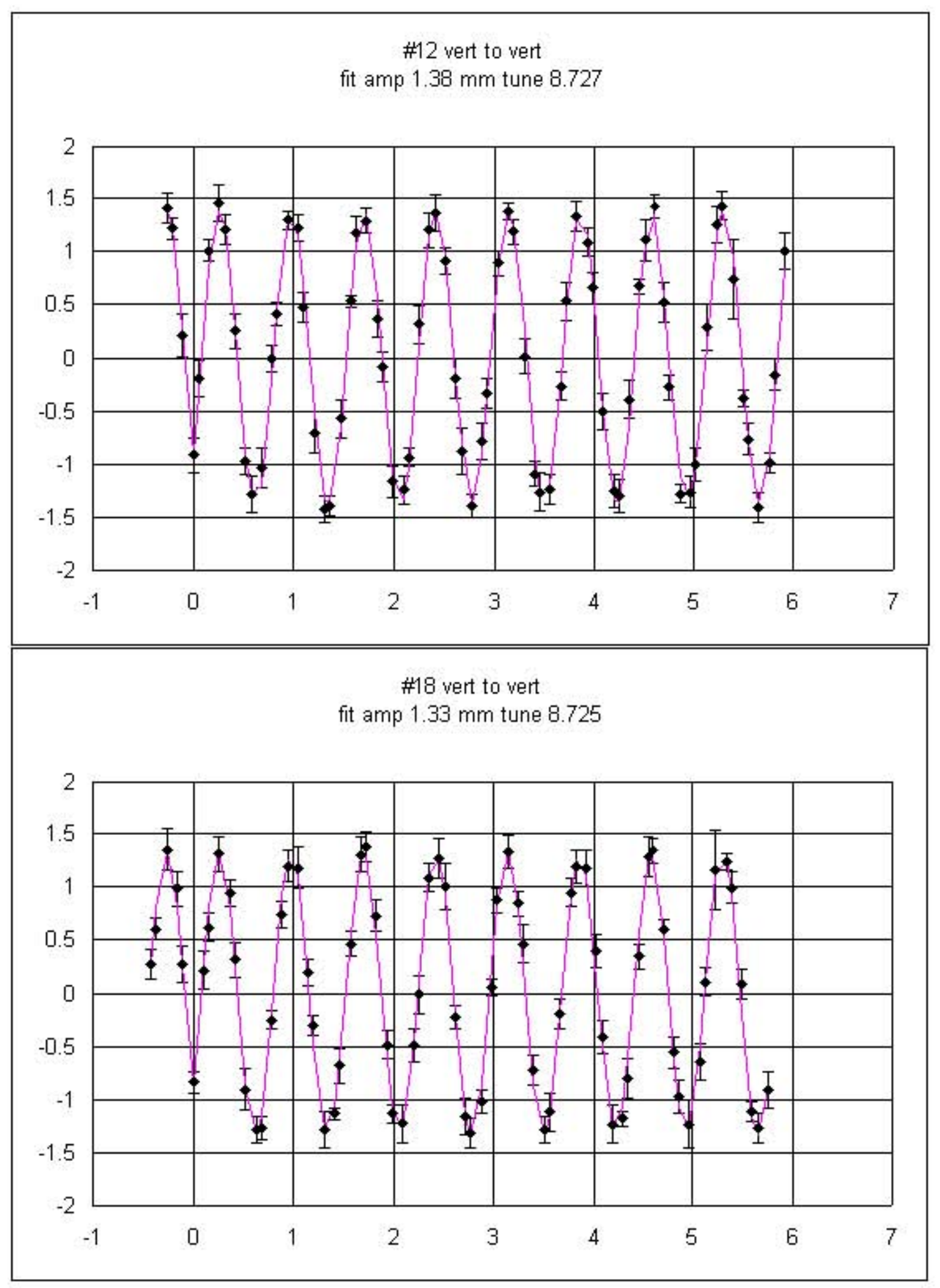


Appendix VII: Horizontal orbit responses to vertical dipoles, gold extraction

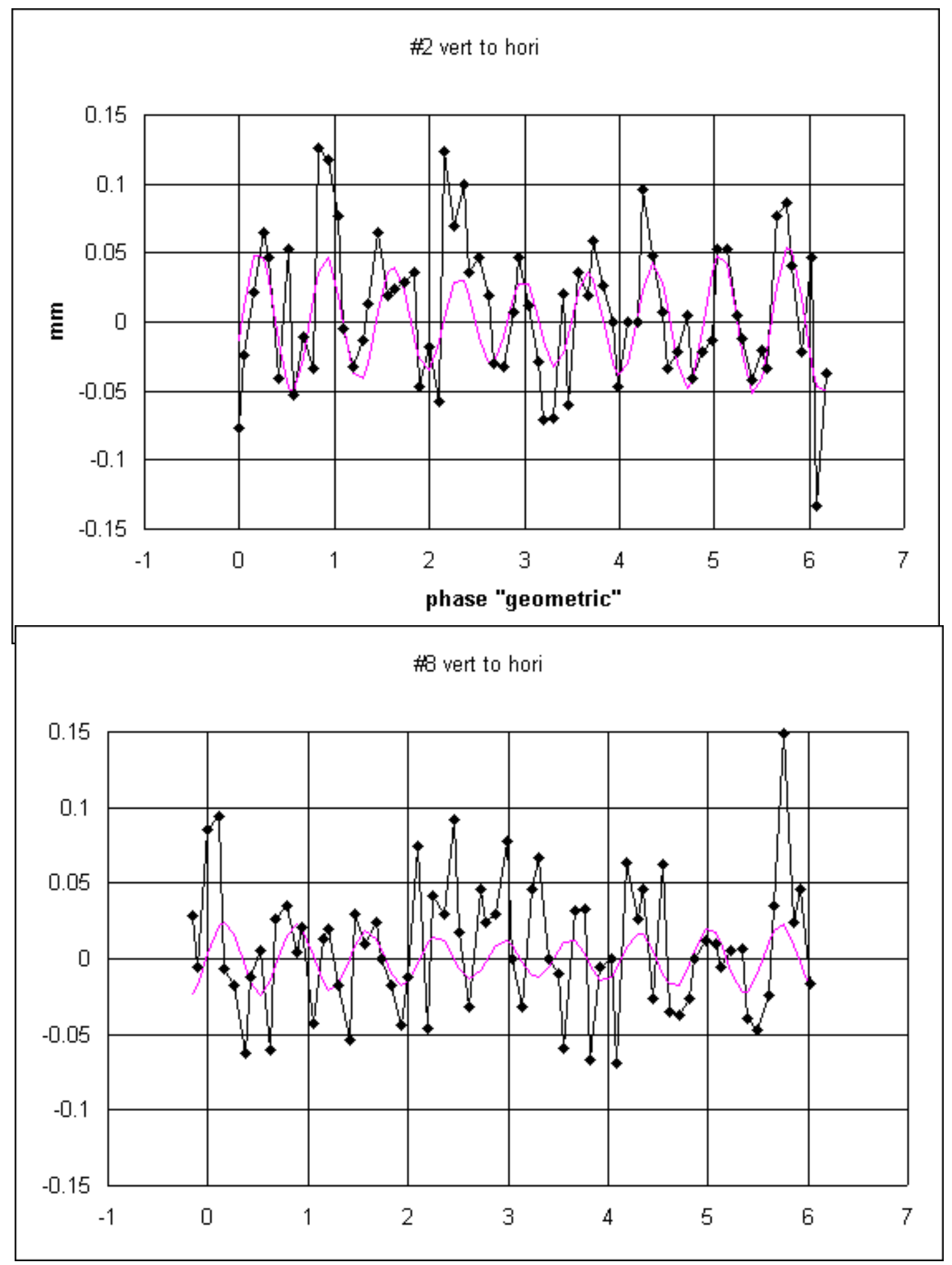




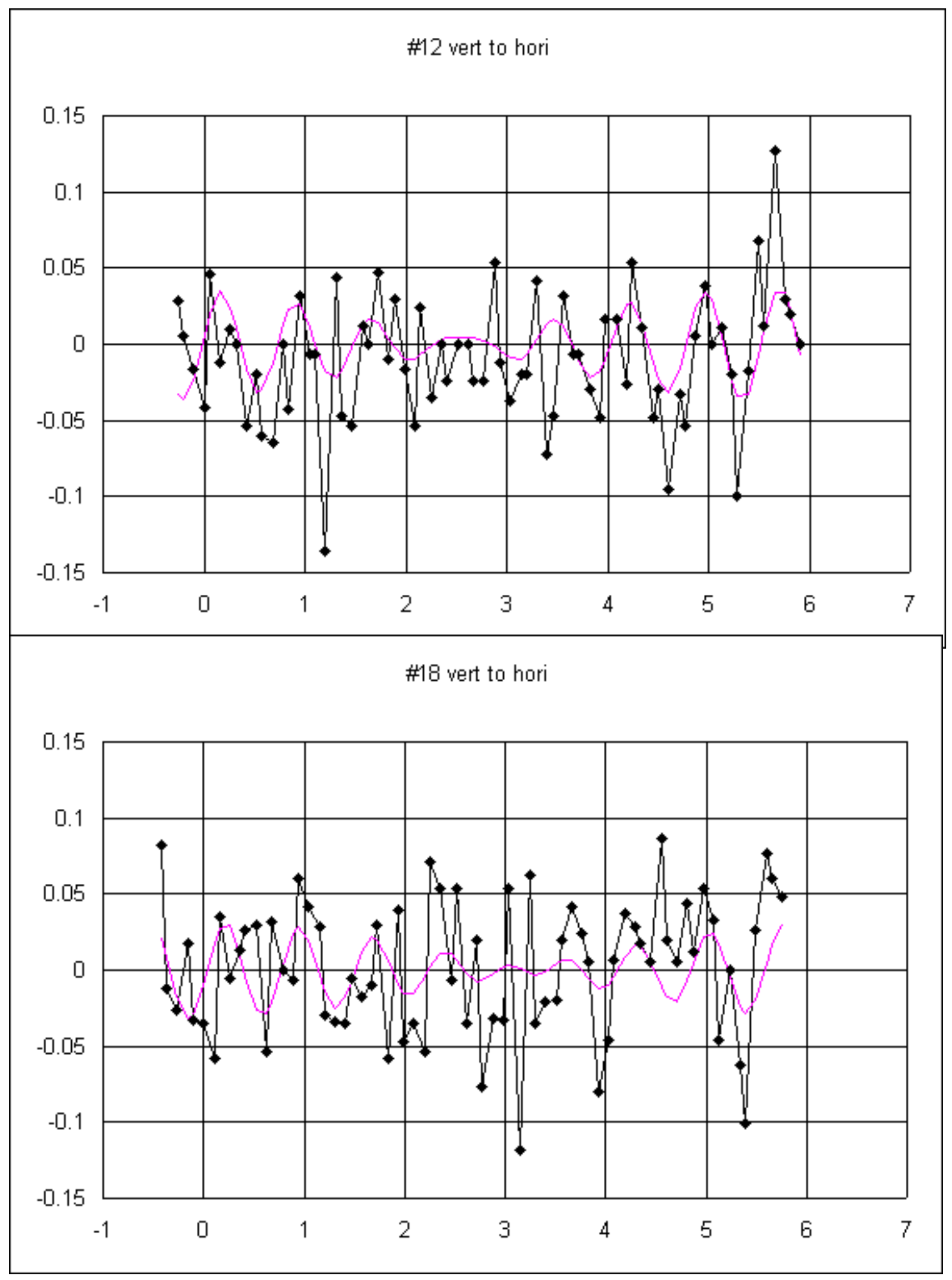


Appendix IX: Experimental investigation of dependence of injection coupling on the amplitude of the current in the exciting dipole

Here we check (using much more recent data) the dependence of ags coupling on kick amplitude, and also the agreement between a tune measurement from the AGS tune meter (now working) and the tune from the simple fitting employed above. Nothing surprising here.
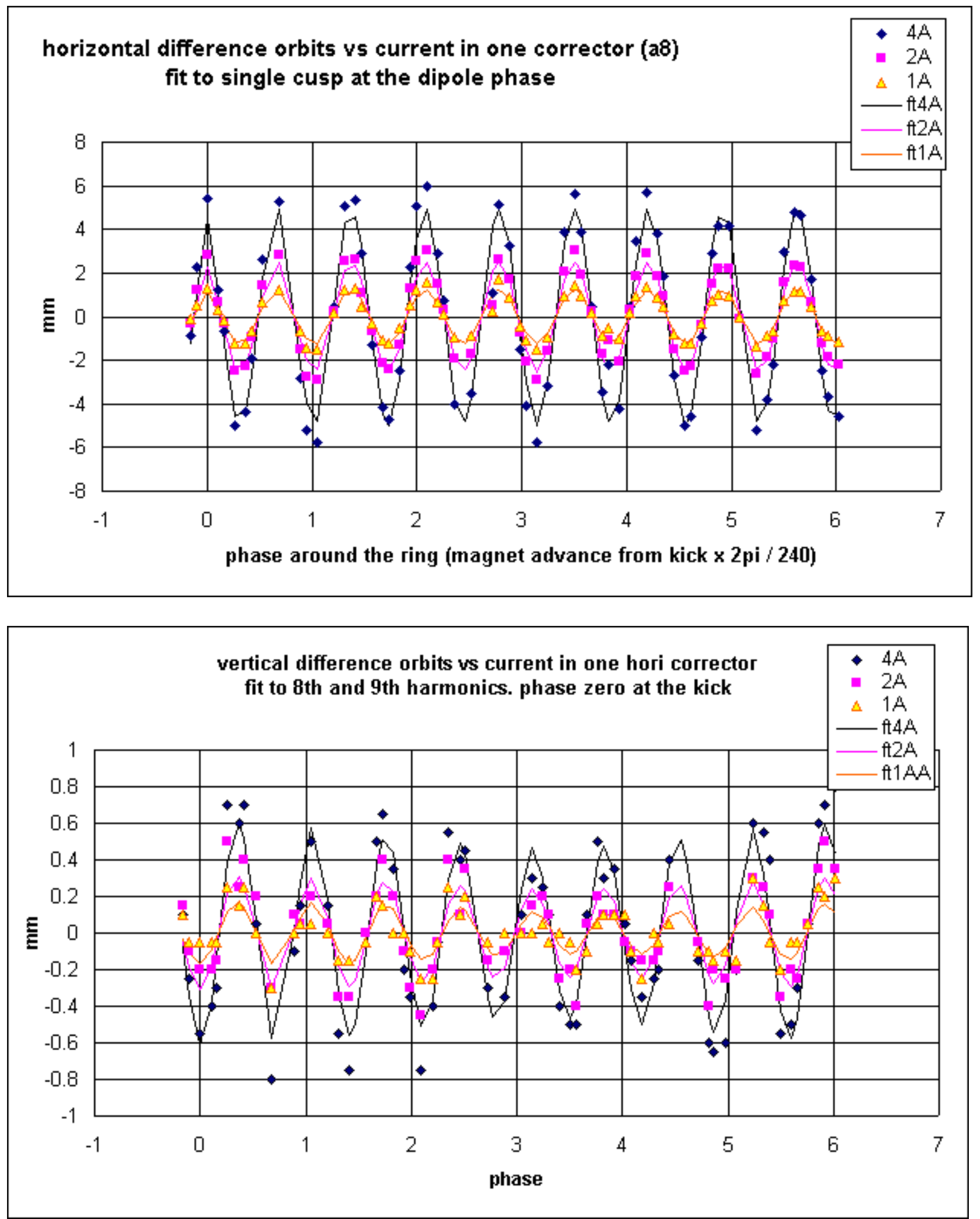
This much more recent set of data was taken soon after the start of the 2010 gold run (11Dec09). The data was taken at $1000 \mathrm{~ms}$ from ags To, so just at end of the injection porch, gold at injection rigidity and at $\mathrm{h}=12$. Here the machine is not "bare", but the skew quads are off. A single corrector dipole, the horizontal at straight section $\mathrm{A} 8$, is powered at $4 \mathrm{~A}$, then $2 \mathrm{~A}$, and then $1 \mathrm{~A}$. The resulting difference orbits are shown. The data is handled similarly to analyses given above. The kicked plane difference orbit is fit to a cusp at the kick with phases taken to be simple geometric in terms of magnets. Amplitude and betatron tune are the fit parameters. In the other (vert) plane the difference orbit is fit to 8th and 9th harmonics. The resulting amplitudes and the tune are given in the final plot. Here there is only the one orbit (i.e. not the "ORM" quality by quantity improvement) and the quality is clearly not as good as with the averages for kicks at every superperiod combined. Nevertheless the results from the fitting indicate that the amplitude of the oscillation in the other plane grows proportional to the amplitude in the kicked plane. Indeed, given the agreement between the planes, it appears that the dipole response is not as linear as it could be. Also the tune from this simple fit agrees reasonably with a measurement from the tune meter FFT- which gives peaks at (Qh, Qv) : (8.684 and 8.816). The fitting (shown below) with 4A - best signal-tonoise -gives Qh of 8.865 .

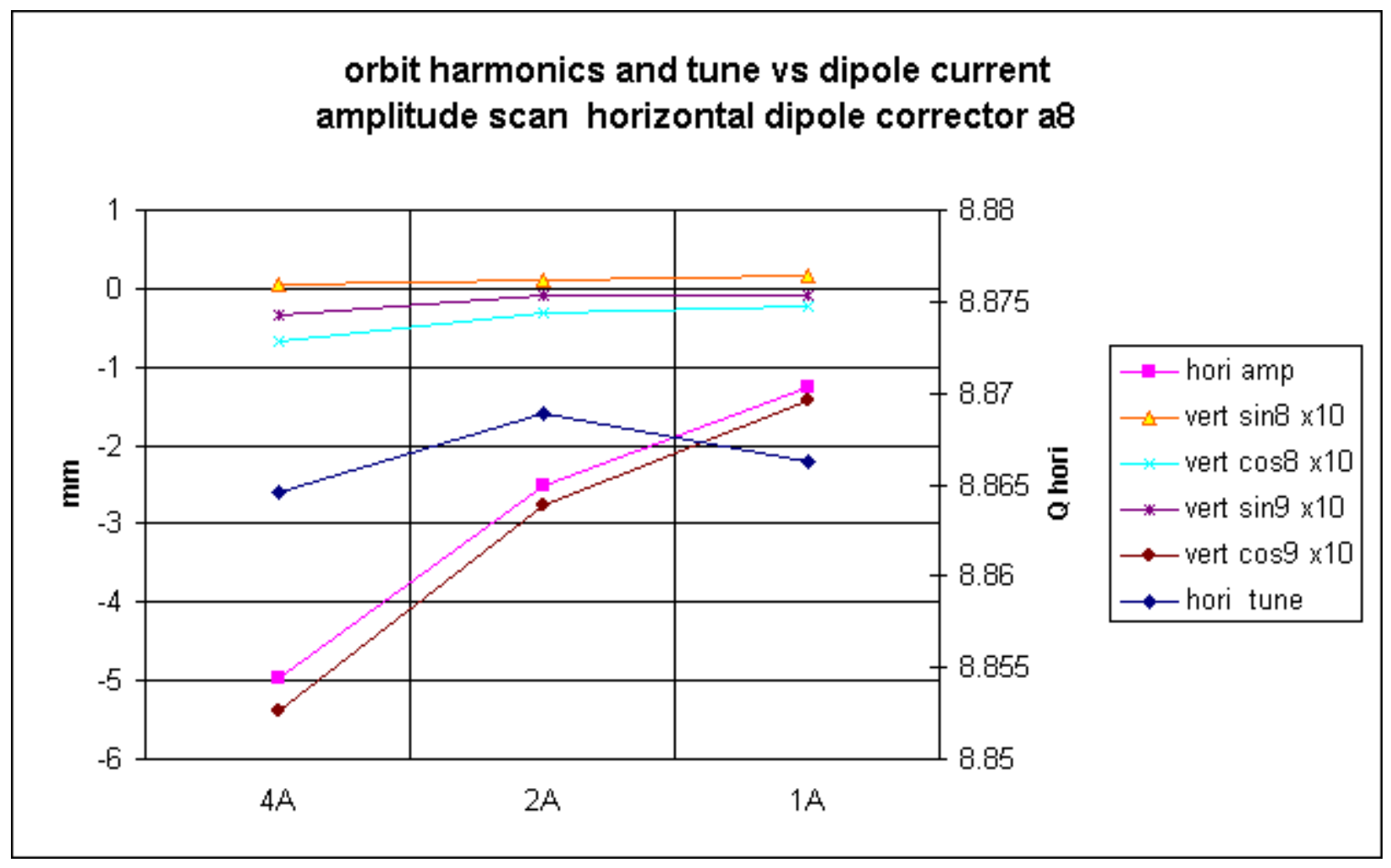

